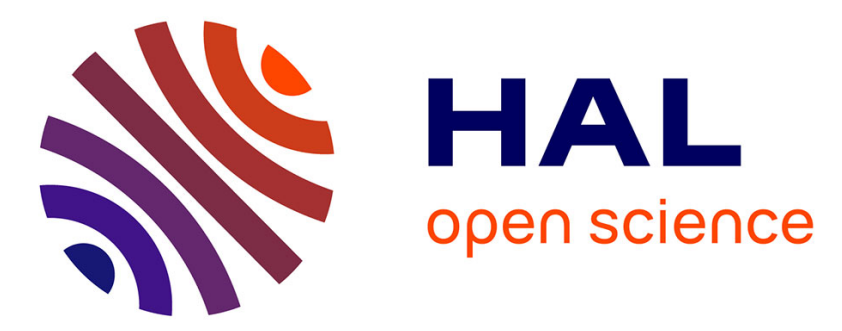

\title{
Velocity-changing collisions in pure H2 and H2-Ar mixture
}

Piotr Wcislo, Ha Tran, Samir Kassi, Alain Campargue, Franck Thibault, Roman Ciurylo

\section{- To cite this version:}

Piotr Wcislo, Ha Tran, Samir Kassi, Alain Campargue, Franck Thibault, et al.. Velocity-changing collisions in pure H2 and H2-Ar mixture. Journal of Chemical Physics, 2014, 141, pp.074301. 10.1063/1.4892414. hal-01065523

\section{HAL Id: hal-01065523 https://hal.science/hal-01065523}

Submitted on 18 Sep 2014

HAL is a multi-disciplinary open access archive for the deposit and dissemination of scientific research documents, whether they are published or not. The documents may come from teaching and research institutions in France or abroad, or from public or private research centers.
L'archive ouverte pluridisciplinaire HAL, est destinée au dépôt et à la diffusion de documents scientifiques de niveau recherche, publiés ou non, émanant des établissements d'enseignement et de recherche français ou étrangers, des laboratoires publics ou privés. 


\title{
Velocity-changing collisions in pure $\mathbf{H}_{2}$ and $\mathbf{H}_{2}$-Ar mixture
}

\author{
P. Wcisło, ${ }^{1, \text { a) }}$ H. Tran, $^{2}$ S. Kassi, ${ }^{3}$ A. Campargue, ${ }^{3}$ F. Thibault, ${ }^{4}$ and R. Ciuryło ${ }^{1}$ \\ ${ }^{1)}$ Institute of Physics, Faculty of Physics, Astronomy and Informatics, Nicolaus Copernicus University, \\ Grudziadzka 5/7, 87-100 Toruń, Poland \\ ${ }^{2)}$ Laboratoire Inter-universitaire des Systèmes Atmosphériques (LISA), CNRS UMR 7583, \\ Université Paris Est Créteil, Université Paris Diderot, Institut Pierre-Simon Laplace, 94010 Créteil Cedex, \\ France \\ ${ }^{3)}$ Laboratoire Interdisciplinaire de Physique, CNRS UMR 5588, Université Joseph Fourier de Grenoble B.P. 87, \\ 38402 Saint-Martin d'Héres Cedex, France \\ 4) Institut de Physique de Rennes, UMR CNRS 6251, Université de Rennes 1, Campus de Beaulieu, Bât.11B, \\ F-35042 Rennes, France
}

(Dated: 22 July 2014)

We show how to effectively introduce a proper description of the velocity-changing collisions into the model of isolated molecular transition for the case of self- and Ar-perturbed $\mathrm{H}_{2}$. We demonstrate that the billiardball (BB) approximation of the $\mathrm{H}_{2}-\mathrm{H}_{2}$ and $\mathrm{H}_{2}$ - $\mathrm{Ar}$ potentials gives an accurate description of the velocitychanging collisions. The BB model results are compared with ab initio classical molecular dynamics simulations (CMDS). It is shown that the BB model correctly reproduces not only the principal properties such as frequencies of velocity-changing collisions or collision kernels, but also other characteristics of $\mathrm{H}_{2}-\mathrm{H}_{2}$ and $\mathrm{H}_{2}$-Ar gas kinetics like rate of speed-changing collisions. Finally, we present line-shape measurement of Q(1) line of the first overtone band of self-perturbed $\mathrm{H}_{2}$. We quantify the systematic errors of line-shape analysis caused by the use of oversimplified description of velocity-changing collisions. These conclusions will have significant impact on recent rapidly developing ultra-accurate metrology based on Doppler-limited spectroscopic measurements like Doppler-width thermometry, atmosphere monitoring, Boltzmann constant determination or transition position and intensity determination for fundamental studies.

PACS numbers: $32.70 . \mathrm{Jz}$

\section{INTRODUCTION}

In recent years significant progress was done in molecular spectroscopy of gas samples. One can note the tremendous increase in accuracy and precision of line shape measurement in the Doppler limited spectroscopy. Let us only mention ultra high sensitivity ${ }^{1,2}$ approaching $10^{-13} \mathrm{~cm}^{-1}$, measurement of transition frequencies ${ }^{3}$ with accuracy at the $\mathrm{kHz}$ level, determination of Boltzmann constant from Doppler line broadening ${ }^{4,5}$ with accuracy approaching $10^{-5}$ and ultra high signal-to-noise ratio ${ }^{6}$ beyond $10^{5}$ level. Such progress is accompanied by the development of line shape theory of isolated spectral line. To give a proper description of the shape of spectral line, two main processes resulting from molecular collision need to be taken into account. The first one is a change of molecule phase or state and the second one is a change of molecule velocity. This paper is devoted to the second process i.e. velocity changes due to collisions, which are responsible for the Dicke narrowing effect. ${ }^{7}$

Semi-analytical models of spectral line shapes ${ }^{8-14}$ allow to include simultaneously basic effects ${ }^{15}$ such as Doppler broadening, Dicke narrowing, ${ }^{7}$ speed-dependent collision broadening and shifting, ${ }^{16}$ correlation between velocity-changing and dephasing or state changing collisions ${ }^{17}$ and nonimpact effects. ${ }^{18} \mathrm{Ab}$ initio line shape

\footnotetext{
a) Electronic mail: piotr.wcislo@fizyka.umk.pl.
}

calculations based on quantum mechanical scattering and solving transport/relaxation equation ${ }^{19-21}$ or based on classical molecular dynamic simulations (CMDS) $)^{22,23}$ can significantly reduce the number of approximations for spectra evaluation.

A realistic description of the velocity-changing collisions is crucial to accurately analyze measured spectra. Moreover velocity-changing collisions are essential for many processes in gases such as thermalization, mass diffusion, heat transfer or viscosity. To these ends, fast and computationally efficient models describing velocity changing collisions are needed. The quality of such models should be verified by comparison with ab initio calculations.

At present, to the best of our knowledge, the most realistic models describing velocity-changing collisions applicable in line shape calculation ${ }^{19,24,25}$ and used for fitting of experimental data ${ }^{25-32}$ are the billiard-ball (BB) model ${ }^{19,33,34}$ and a bit more general Blackmore model, ${ }^{19,35}$ which assumes repulsive inverse-power potential. The most important advantage of the $\mathrm{BB}$ model is that it allows to take into account the speed-dependence of frequency of velocity-changing collisions as well as the speed- and direction-changing collisions for a given perturber/absorber mass ratio in a proper way.

The aim of this paper is to show that the BB approximations of real $\mathrm{H}_{2}-\mathrm{H}_{2}$ and $\mathrm{H}_{2}$-Ar collision potentials lead to appropriate kinetic properties. We check it by referring the results obtained from the $\mathrm{BB}$ model to those from $a b$ initio classical molecular dynamic simulations 
(CMDS). To compare the $\mathrm{H}_{2}-\mathrm{H}_{2}$ and $\mathrm{H}_{2}$-Ar gases kinetics, we directly compared the collision kernels resulting from the BB model and from CMDS. Moreover we developed an operator formalism allowing evaluation of the autocorrelation function for different molecule velocityrelated quantities for any model of velocity-changing collisions, see Sec. III. We used it to compare the relaxations of kinetic properties obtained from the BB model with those from CMDS.

Finally, the BB model was confronted with the selfbroadened shape of the $\mathrm{Q}(1)$ line of the first overtone band of $\mathrm{H}_{2}$ measured using a cavity ring-down spectroscopy $(\mathrm{CRDS})^{1}$ system. We quantified the systematic errors in molecular spectra analysis caused by use of oversimplified phenomenological models of velocitychanging collisions like the hard- and soft-collision $\left(\mathrm{SC}^{36}\right.$ and $\mathrm{HC}^{37}$, respectively) models often used in spectral line shapes analysis. ${ }^{17,38-45}$. These results allow to remove the limitations on accuracy of physical quantities obtained from spectra analysis and therefore they are important for optical metrology based on molecular spectroscopy like Doppler-width thermometry and Boltzmann constant determination, ${ }^{4,5,46}$ atmosphere monitoring, ${ }^{47,48}$ or transition position and intensity determination for fundamental studies. ${ }^{49}$

\section{DESCRIPTION OF VELOCITY-CHANGING COLLISIONS}

Intermolecular collisions in gas phase lead to the change of the molecule initial velocity $\vec{v}_{0}$. This phenomenon can be investigated using the probability $P\left(\vec{v}, t ; \vec{v}_{0}\right)$ that the molecule having the velocity $\vec{v}_{0}$ at $t=0$ will have the velocity $\vec{v}$ at time $t .50,51$ The time evolution of this probability is determined by the velocitychanging collision operator $\hat{S}_{\mathrm{VC}}$ describing intermolecular collisions in gas and can be found by solving the following integro-differential equation

$$
\frac{\partial}{\partial t} P\left(\vec{v}, t ; \vec{v}_{0}\right)=\hat{S}_{\mathrm{VC}} P\left(\vec{v}, t ; \vec{v}_{0}\right)
$$

with the initial condition $P\left(\vec{v}, 0 ; \vec{v}_{0}\right)=\delta^{3}\left(\vec{v}-\vec{v}_{0}\right)$. The collision operator $\hat{S}_{\mathrm{VC}}$ from Eq. (1) can be expressed in terms of the collision kernel $f\left(\vec{v} \leftarrow \vec{v}^{\prime}\right)$ describing proba- bility rates of velocity-change from $\vec{v}^{\prime}$ to $\vec{v}$

$$
\begin{aligned}
& \hat{S}_{\mathrm{VC}} P\left(\vec{v}, t ; \vec{v}_{0}\right) \\
& =\int d^{3} \vec{v}^{\prime}\left[f\left(\vec{v} \leftarrow \vec{v}^{\prime}\right) P\left(\vec{v}^{\prime}, t ; \vec{v}_{0}\right)-f\left(\vec{v}^{\prime} \leftarrow \vec{v}\right) P\left(\vec{v}, t ; \vec{v}_{0}\right)\right] .
\end{aligned}
$$

It is also convenient ${ }^{19,33,52,53}$ to introduce the operator $\hat{S}_{\mathrm{VC}}^{f}$ which is related to the operator $\hat{S}_{\mathrm{VC}}$ in the following way

$$
\hat{S}_{\mathrm{VC}} f_{m}(\vec{v}) h(\vec{v})=f_{m}(\vec{v}) \hat{S}_{\mathrm{VC}}^{f} h(\vec{v}) .
$$

Here, $h(\vec{v})$ is some function of $\vec{v}$ and $f_{m}(\vec{v})=$ $\left(\pi v_{m}^{2}\right)^{-3 / 2} \exp \left(-v^{2} / v_{m}^{2}\right)$ is the Maxwellian distribution of the investigated molecule velocity $\vec{v}, v_{m}=\sqrt{2 k_{B} T / m_{1}}$ is the most probable speed of this molecule, $m_{1}$ being its molecular mass, $k_{B}$ is the Boltzmann constant, $T$ the gas temperature.

One of the simplest model describing the velocitychanging collisions is a $\mathrm{HC}$ model ${ }^{37,39}$ assuming that the molecule velocity is totally thermalized after each collision. The collision kernel associated with this model is given by a simple analytical formula

$$
f_{\mathrm{HC}}\left(\vec{v} \leftarrow \vec{v}^{\prime}\right)=\nu_{\mathrm{diff}} f_{m}(\vec{v}),
$$

where $\nu_{\text {diff }}$ is an effective frequency of velocity-changing collisions, see Appendix A. This simple model does not originate from any particular description of binary collisions therefore we treat it here as a phenomenological approximation to a proper description of velocity-changing collisions. Note that the $\mathrm{HC}$ model should not be attributed to the case of light molecules diluted in infinitely heavier perturbers bath. In such a case only the velocity direction is thermalized, while the molecule speed remains unchanged under the collision. For more details see Refs. 24 and 27, and Appendix A.

A more advanced model describing velocity-changing collisions, which allows to properly incorporate the mass ratio of colliding pair is the so called billiard-ball model or rigid-spheres model. ${ }^{19,33,34}$ In this approach, the molecular potential is modelled by considering only the repulsive wall of two rigid spheres having masses $m_{1}$ and $m_{2}$. The collision kernel for the BB model can be expressed analytically following Liao, Bjorkholm, and Berman ${ }^{34}$

$$
\begin{aligned}
& f_{\mathrm{BB}}\left(\vec{v} \leftarrow \vec{v}^{\prime}\right)=\nu^{(0)} \frac{1}{v_{m}^{2}} \frac{3}{32 \pi} \frac{\sqrt{1+\alpha}(1+\alpha)^{2}}{\alpha^{2}} \frac{1}{\sqrt{v^{2}-2 v v^{\prime} \cos \theta+v^{\prime 2}}} \times \\
& \times \exp \left(-\frac{(1-\alpha)^{2}}{4 \alpha} \frac{v^{\prime 2}}{v_{m}^{2}}-\frac{(1+\alpha)^{2}}{4 \alpha} \frac{v^{2}}{v_{m}^{2}}-\frac{(\alpha+1)(\alpha-1)}{2 \alpha} \frac{v v^{\prime}}{v_{m}^{2}} \cos \theta+\frac{\alpha v^{2} v^{\prime 2} \sin ^{2} \theta}{v_{m}^{2}\left(v^{2}-2 v v^{\prime} \cos \theta+v^{\prime 2}\right)}\right),
\end{aligned}
$$

where $\theta$ is the angle between $\vec{v}$ and $\vec{v}^{\prime}$,

$$
\nu^{(0)}=(8 / 3) \sqrt{\pi}\left(\alpha^{-1}+1\right)^{-1 / 2} v_{m} n_{2} d^{2}
$$

is the first-order effective frequency of velocity-changing 
collisions, see also Appendix A and Refs. 24 and 33, $\alpha=m_{2} / m_{1}$ is the perturber to investigated molecule mass ratio, $d$ is the mean hard-sphere diameter of the colliding particles and $n_{2}$ is the density number of perturbers. This kernel was applied to studies of velocitychanging collisions in two-photon spectroscopy. ${ }^{34}$

The matrix elements of the collision operator $\hat{S}_{\mathrm{VC}}^{f}$ for the $\mathrm{BB}$ model in the basis of the Burnett function can be evaluated from analytical expressions given by Lindenfeld and Shizgal. ${ }^{54}$ This operator was used for the study of Dicke narrowed spectral lines. ${ }^{19,24}$

Collision kernels can be also derived from CMDS as done in Refs. 51 and 55 where details of calculations can be found. From an interaction potential, the time evolution of a large number of molecules can be computed using classical mechanics. ${ }^{56}$ For the present study, CMDS have been performed as follows. The molecules are initially randomly placed inside a cubic box with the constraint that they should be separated from each other by distances at which the interaction potential is negligible, thus forbidding any unphysical situations involving strong intermolecular interactions at time zero. Several hundred of boxes with periodic boundary conditions have been used, each contains about 5000 molecules (more in the case of mixture). The size of each box is deduced from the perfect gas law, with the temperature and density used in the calculation. For the velocities, speeds verifying a Maxwell-Boltzmann distribution have been randomly attributed to the molecules while random values have been chosen for velocity-orientations. At each time step, the central force applied to each molecule by its neighbors is computed. The velocity and hence the position of the molecules are then determined at each time step. A temporization time has been observed in order to ensure that the initial configuration is completely thermalized..$^{56}$

The velocity modulus and orientation have been discretized into intervals in which each molecule has been labelled. The time evolution of the system has been then followed by considering, at each time step, the fraction of the molecules having an initial velocity $\vec{v}_{0}$ (whose modulus is in one speed interval $\Delta v_{i}$ ) which have a velocity $\vec{v}$ whose modulus is in another interval $\left(\Delta v_{j}\right)$ and making an angle $\theta(t)$ (within an interval $\Delta \theta_{k}$ ) with respect to $\vec{v}_{0}$. This quantity is directly related to the conditional probability $P\left(\vec{v}, t ; \vec{v}_{0}\right)$. As shown in Ref. 51 , from the slopes of the conditional probabilities at time zero, one can directly determine the collision kernels $f_{\mathrm{CMDS}}\left(\vec{v} \leftarrow \vec{v}^{\prime}\right)$.

\section{AUTOCORRELATION FUNCTION}

Important information about relaxation of molecule velocity, speed or direction of motion caused by collisions in gas can be found using the concept of the autocorrelation function. ${ }^{57}$ The autocorrelation function $\Phi_{g(\vec{v})}(t)$ for some quantity $g(\vec{v})$ dependent of the molecule velocity can be defined as the following statistical average:

$$
\begin{aligned}
& \Phi_{g(\vec{v})}(t)=\langle g[\vec{v}(t)] g[\vec{v}(0)]\rangle_{\text {Stat.Av. }}= \\
& =\int d^{3} \vec{v} \int d^{3} \overrightarrow{v_{0}} g(\vec{v}) P\left(\vec{v}, t ; \vec{v}_{0}\right) g\left(\vec{v}_{0}\right) f_{m}\left(\vec{v}_{0}\right)= \\
& =\int d^{3} \vec{v} g(\vec{v}) \phi_{g(\vec{v})}(\vec{v}, t),
\end{aligned}
$$

where

$$
\phi_{g(\vec{v})}(\vec{v}, t)=\int d^{3} \overrightarrow{v_{0}} P\left(\vec{v}, t ; \vec{v}_{0}\right) g\left(\vec{v}_{0}\right) f_{m}\left(\vec{v}_{0}\right) .
$$

It can be shown, by multiplying both sides of Eq. (1) by $g\left(\vec{v}_{0}\right) f_{m}\left(\vec{v}_{0}\right)$ and integrating it over the initial velocity $\vec{v}_{0}$, that the function $\phi_{g(\vec{v})}(\vec{v}, t)$ also fulfils the kinetic equation, Eq. (1)

$$
\frac{\partial}{\partial t} \phi_{g(\vec{v})}(\vec{v}, t)=\hat{S}_{\mathrm{VC}} \phi_{g(\vec{v})}(\vec{v}, t)
$$

with the initial condition $\phi_{g(\vec{v})}(\vec{v}, 0)=g(\vec{v}) f_{m}(\vec{v})$. The function $\phi_{g(\vec{v})}(\vec{v}, t)$ can be expressed in terms of a function $h_{g(\vec{v})}(\vec{v}, t)$ as $\phi_{g(\vec{v})}(\vec{v}, t)=h_{g(\vec{v})}(\vec{v}, t) f_{m}(\vec{v})$. Using Eq. (3), the kinetic equation, Eq. (9), can be transformed to

$$
\frac{\partial}{\partial t} h_{g(\vec{v})}(\vec{v}, t)=\hat{S}_{\mathrm{VC}}^{f} h_{g(\vec{v})}(\vec{v}, t),
$$

which is analogous to Eq. (9), but with the operator $\hat{S}_{\mathrm{VC}}^{f}$ instead of $\hat{S}_{\mathrm{VC}}$. In this case the initial condition for the function $h_{g(\vec{v})}(\vec{v}, t)$ should be taken as $h_{g(\vec{v})}(\vec{v}, 0)=g(\vec{v})$. The autocorrelation function $\Phi_{g(\vec{v})}(t)$ can be given by the following expression

$$
\Phi_{g(\vec{v})}(t)=\int d^{3} \vec{v} f_{m}(\vec{v}) g(\vec{v}) h_{g(\vec{v})}(\vec{v}, t)
$$

using the introduced function $h_{g(\vec{v})}(\vec{v}, t)$.

Having given the collision operator $\hat{S}_{\mathrm{VC}}^{f}$, the autocorrelation function can be calculated using its eigenvalues $\lambda_{n}$ and its unity normalized, $\int d^{3} \vec{v} f_{m}(\vec{v}) e_{n}(\vec{v}) e_{n^{\prime}}(\vec{v})=\delta_{n, n^{\prime}}$, eigenfunctions $e_{n}(\vec{v})$, enumerated by discreet index $n$, which fulfil standard eigenequation

$$
\hat{S}_{\mathrm{VC}}^{f} e_{n}(\vec{v})=\lambda_{n} e_{n}(\vec{v}) .
$$

It follows from Eqs. (10) and (12) that each eigenfunction $e_{n}(\vec{v})$ will decay with its characteristic rate given by the corresponding eigenvalue $\lambda_{n}$. Hence the solution of Eq. (10) can be obtained in the form of series

$$
h_{g(\vec{v})}(\vec{v}, t)=\sum_{n=0}^{\infty} a_{n} e^{\lambda_{n} t} e_{n}(\vec{v})
$$

where

$$
a_{n}=\int d^{3} \vec{v} f_{m}(\vec{v}) g(\vec{v}) e_{n}(\vec{v}) .
$$


Inserting Eq. (13) into Eq. (11), the following series for the autocorrelation function is obtained

$$
\Phi_{g(\vec{v})}(t)=\sum_{n=0}^{\infty} a_{n}^{2} e^{\lambda_{n} t}
$$

The expression given above was used to calculate the autocorrelation function for the BB model but can be easily applied in other cases in which the matrix elements of $\hat{S}_{\mathrm{VC}}^{f}$ in some set of basis function are known. Simple analytical forms of Eq. (15) for the SC and HC cases are given in Appendix B.

The calculations of the autocorrelation function, in case of molecular dynamic simulations, are performed by applying the definition given by Eq. (7) in the appropriate form for limited sample in the statistical average ${ }^{51}$

$$
\Phi_{g(\vec{v})}(t)=\frac{1}{N} \sum_{i=0}^{N} g\left[\vec{v}_{i}(t)\right] g\left[\vec{v}_{i}(0)\right] .
$$

Here $\vec{v}_{i}(t)$ is the time-dependent velocity of $i$-th traced molecule and $N$ is the number of molecule traces used for the average in Eq. (16).

\section{INTERACTION POTENTIALS AND ASSUMED PHYSICAL CONDITIONS}

Isotropic Lennard-Jones potentials $V(r)=$ $4 \varepsilon\left[(\sigma / r)^{12}-(\sigma / r)^{6}\right]$ describing the $\mathrm{H}_{2}-\mathrm{H}_{2}$ and $\mathrm{H}_{2}$ Ar interactions are presented in the Fig. 1 (b) and (c), respectively, as black solid lines. Parameters of these potentials were taken from Ref. 58 (for $\mathrm{H}_{2}-\mathrm{H}_{2}$ : $\varepsilon_{\mathrm{H}_{2}-\mathrm{H}_{2}} / k_{B}=33.50 \mathrm{~K}, \sigma_{\mathrm{H}_{2}-\mathrm{H}_{2}}=2.93 \AA$ and for $\mathrm{H}_{2}-$ Ar: $\left.\varepsilon_{\mathrm{H}_{2}-\mathrm{Ar}} / k_{B}=64.8 \mathrm{~K}, \sigma_{\mathrm{H}_{2}-\mathrm{Ar}}=3.16 \AA\right)$. These potentials were directly used for CMDS. Within the $\mathrm{BB}$ model the mean diameters of the hard spheres related to $\mathrm{H}_{2}-\mathrm{H}_{2}$ and $\mathrm{H}_{2}$-Ar colliding molecules were chosen such that the hard-spheres wall intersects the Lennard-Jones potentials at the mean collisional energy $\left(\bar{\epsilon} / k_{B}=(3 / 2) T=444 \mathrm{~K}\right.$ at $\left.T=296 \mathrm{~K}\right)$, see blue solid lines in Fig. 1 (b) and (c). Consequently, the mean hard-sphere diameters of colliding particles for $\mathrm{H}_{2}-\mathrm{H}_{2}$ and $\mathrm{H}_{2}$-Ar are $d_{\mathrm{H}_{2}-\mathrm{H}_{2}}=2.53 \AA$ and $d_{\mathrm{H}_{2}-\mathrm{Ar}}=2.84 \AA$, respectively. As a reference, the Maxwellian distribution of collision energy is shown in Fig. 1 (a) for $T=296 \mathrm{~K}$. The level of the energy corresponding to the most probable speed $(296 \mathrm{~K})$ and the level of the mean energy $(444 \mathrm{~K})$ are indicated by orange and red dashed lines, respectively.

The real $\mathrm{H}_{2}-\mathrm{H}_{2}$ and $\mathrm{H}_{2}$ - $\mathrm{Ar}$ potentials are almost isotropic and in the description of the velocity-changing collisions, even at high level of experimental spectra accuracy, the anisotropic terms can be neglected. Note, however, that the process of phase/state changing under collision, which is out of the scope of this paper, is very sensitive to the anisotropic part of the potential and to describe it, a full potential energy surface (PES) should be considered.

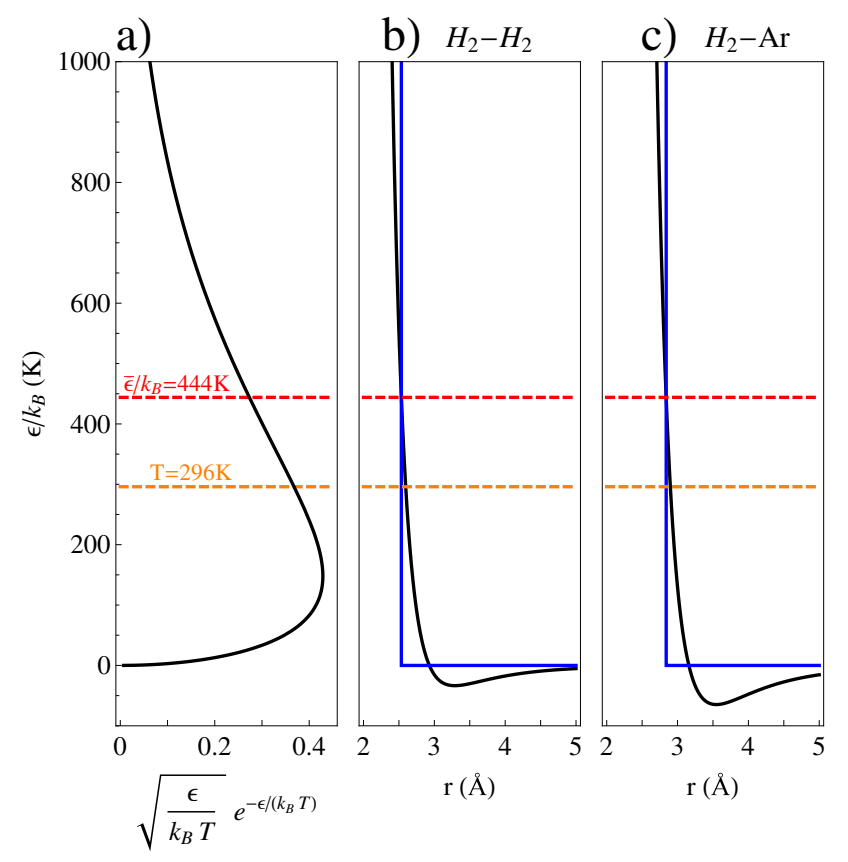

FIG. 1. (Color online) (a) Maxwell-Boltzmann collision energy distribution for $T=296 \mathrm{~K}$. (b) and (c) Isotropic Lennard-Jones potential for colliding $\mathrm{H}_{2}-\mathrm{H}_{2}$ and $\mathrm{H}_{2}$-Ar, respectively. The hard-sphere potentials, illustrated by blue lines, were constructed so that they intersect the LennardJones curves at the mean collision energy $\left(\bar{\epsilon} / k_{B}=444 K\right)$. The mean hard-sphere diameters for $\mathrm{H}_{2}-\mathrm{H}_{2}$ and $\mathrm{H}_{2}$ - $\mathrm{Ar}$ are $d_{\mathrm{H}_{2}-\mathrm{H}_{2}}=2.53 \AA$ and $d_{\mathrm{H}_{2}-\mathrm{Ar}}=2.84 \AA$, respectively.

In the following calculations we assumed that the masses of $\mathrm{H}_{2}$ and $\mathrm{Ar}$ are $m_{H_{2}}=2.016 \mathrm{u}$ and $m_{A r}=$ $39.96 \mathrm{u}$, respectively. ${ }^{59}$

\section{RESULTS AND DISCUSSION}

\section{A. $\mathbf{H}_{2}-\mathbf{H}_{2}$ collisions}

One of the most fundamental parameter describing the velocity-changing collisions is the effective frequency of these collisions $\nu_{\text {diff }}$ related to the diffusion coefficient $D$, see Appendix A. The first-order approximation to $\nu_{\text {diff }}$ within the $\mathrm{BB}$ model, is given by Eq. (6). For the $\mathrm{H}_{2}$ $\mathrm{H}_{2}$ system with the mass ratio $\alpha=1$ and the mean hard-sphere diameters $d_{\mathrm{H}_{2}-\mathrm{H}_{2}}=2.53 \AA$ in the hydrogen density $n_{\mathrm{H}_{2}}=1 \mathrm{amg}$ and temperature $T=296 \mathrm{~K}$ the following $\nu_{\mathrm{H}_{2}-\mathrm{H}_{2}}^{(0)}=1 /(111.34 \mathrm{ps})$ is obtained. The value of $\nu_{\mathrm{diff}, \mathrm{H}_{2}-\mathrm{H}_{2}}=\nu_{\mathrm{H}_{2}-\mathrm{H}_{2}}^{(0)} / f_{D}=1 /(113.45 \mathrm{ps})$ is obtained using a technique presented by Lindenfeld, ${ }^{33}$ see 
also Ref. 24. The $f_{D}$ factor for the BB model depends only on the mass ratio and $f_{D} \approx 1.018954$ for $\alpha=1$.
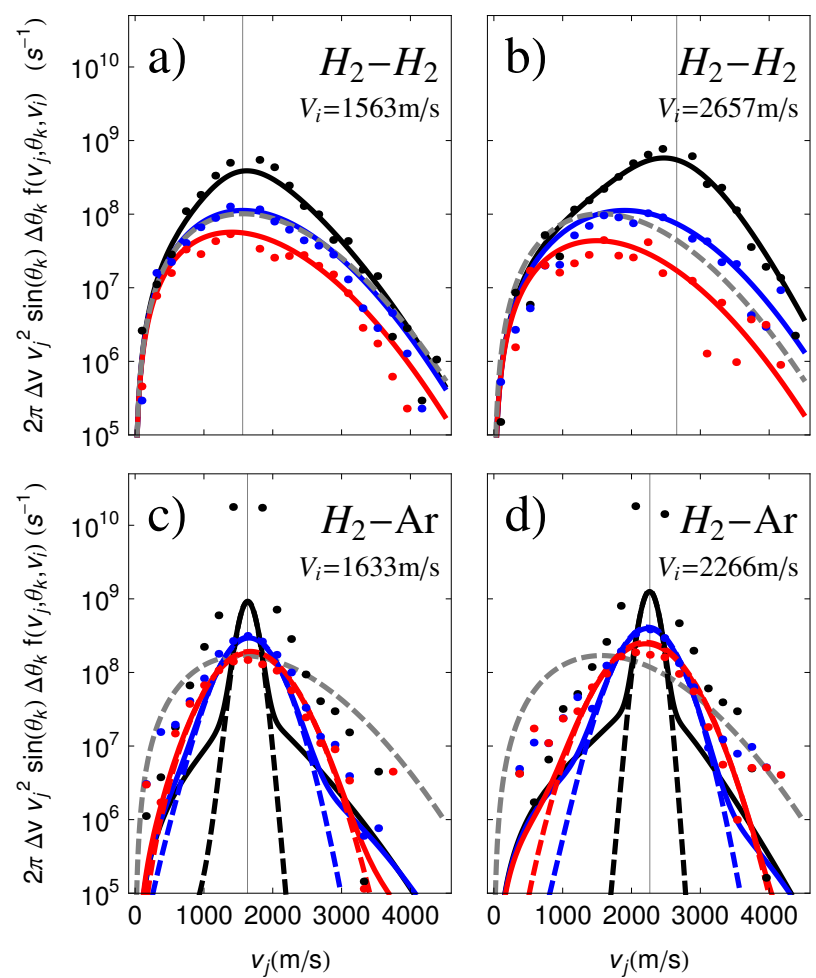

FIG. 2. (Color online) (a) and (b) Collision kernel for $\mathrm{H}_{2}$ $\mathrm{H}_{2}$ for $T=296 \mathrm{~K}$ and $n_{\mathrm{H}_{2}}=1 \mathrm{amg}$. Dots correspond to kernel obtained from $\mathrm{CMDS}^{51}$ and solid lines to kernel from BB model. Black, blue and red colors designate the results for $\theta_{k}=36^{\circ}, 90^{\circ}$ and $144^{\circ}$, respectively. Charts (a) and (b) were plotted for $v_{i}=1563 \mathrm{~m} / \mathrm{s}$ and for $v_{i}=2657 \mathrm{~m} / \mathrm{s}$, respectively. For comparison, the HC kernel with the effective frequency of velocity-changing collisions $\nu_{\text {diff }}=1 / \tau_{\vec{v}}^{\text {CMDS }}=$ $1 /(113.4 \mathrm{ps})=8.82 \times 10^{9} \mathrm{~s}^{-1}$ is plotted as a gray dashed line. The $\theta_{k}$ angle element was $\sin \theta_{k} \Delta \theta_{k}=\Delta\left(-\cos \theta_{k}\right)=0.2$ and the speed element $\Delta v=214.096 \mathrm{~m} / \mathrm{s}$.

(c) and (d) Collision kernel for $\mathrm{H}_{2}$-Ar for $T=296 \mathrm{~K}$. Dots and solid lines correspond to kernels for a mixture of $0.05 \mathrm{amg}$ of $\mathrm{H}_{2}$ and 0.95 amg of Ar obtained from CMDS and BB, respectively. The dashed lines correspond to the BB kernel for the case of $\mathrm{H}_{2}$ infinitely diluted in $\operatorname{Ar}\left(n_{\mathrm{Ar}}=1 \mathrm{amg}, n_{\mathrm{H}_{2}} \ll n_{\mathrm{Ar}}\right)$. Black, blue and red colors designate the $\theta_{k}=25.84^{\circ}, 84.26^{\circ}$ and $154.16^{\circ}$ angles, respectively. (c) and (d) panel correspond to $v_{i}=1633 \mathrm{~m} / \mathrm{s}$ and $v_{i}=2266 \mathrm{~m} / \mathrm{s}$, respectively. The HC kernel with the effective frequency of velocity-changing collisions $\nu_{\text {diff }}=1 / \tau_{\vec{v}}^{\text {CMDS }}=1 /(65.8 \mathrm{ps})=15.20 \times 10^{9} \mathrm{~s}^{-1}$ is plotted as a gray dashed line. The $\theta_{k}$ angle element was $\sin \theta_{k} \Delta \theta_{k}=\Delta\left(-\cos \theta_{k}\right)=0.2$ and the speed element $\Delta v=210.962 \mathrm{~m} / \mathrm{s}$.

The effective frequency of velocity-changing collisions gives only an average measure of collisional properties. Much deeper description can be deduced from the form of the collision kernels, which give the rate of the molecule velocity change from $\vec{v}_{i}$ before the collision to $\vec{v}_{j}$ after the collision. In the following text, we denote the angle between $\vec{v}_{i}$ and $\vec{v}_{j}$ by $\theta_{k}$. The comparison between kernels obtained with CMDS and the BB model is shown in Fig. 2 (a) and (b). Both kernels are almost the same within the CMDS numerical noise, what demonstrates that the hard-sphere approximation gives a very good description of $\mathrm{H}_{2}-\mathrm{H}_{2}$ collisions. Note that these two calculations originate from the same $\mathrm{H}_{2}-\mathrm{H}_{2}$ potential, see Fig. 1, and no parameter was adjusted. In Fig. 2, for comparison, we have also plotted the collision kernel for the HC model (gray dashed line) given by Eq. (4). This kernel does not depend on the $\theta_{k}$ angle and coincides with $a b$ initio CMDS kernel only for $\theta_{k} \approx 90^{\circ}$. This essential difference between the $\mathrm{HC}$ and $a b$ initio CMDS kernels is the principal physical reason explaining why profiles originating from $\mathrm{HC}$ model ${ }^{17,39,43,44}$ are not able to fully reproduce the high quality molecular spectra of self-perturbed hydrogen, see Sec. VI. Note that the effective frequency of the velocity-changing collisions in the $\mathrm{HC}$ model was set to match the relaxation time of velocity in CMDS, see discussion later on.
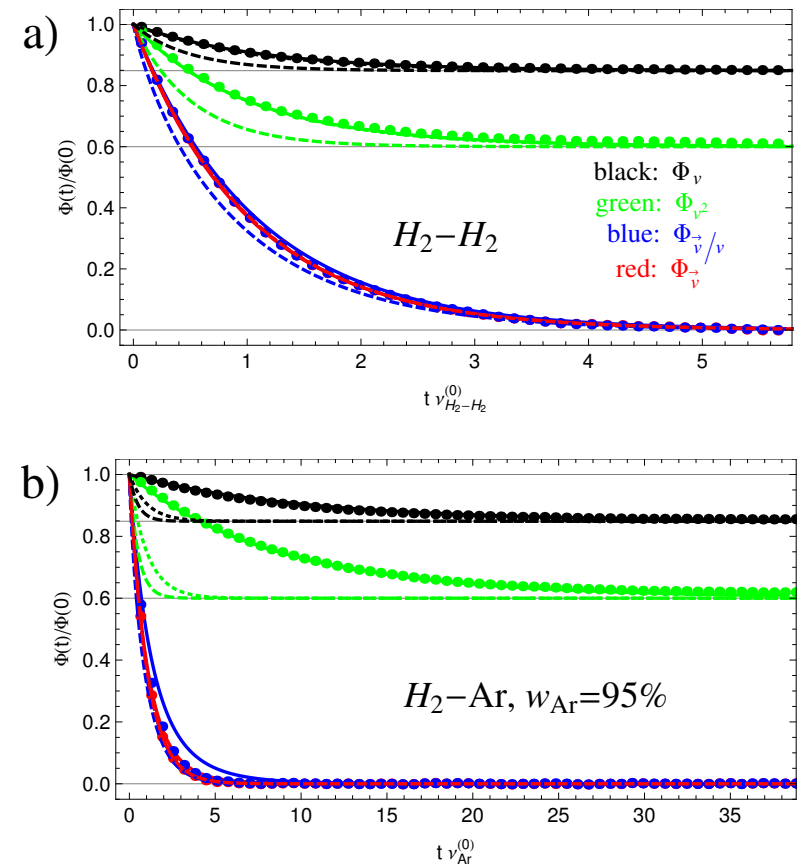

FIG. 3. (Color online) Normalized autocorrelation functions (a) for self perturbed $\mathrm{H}_{2}$ and (b) for $\mathrm{H}_{2}$ perturbed by $\mathrm{Ar} / \mathrm{H}_{2}$ mixture (95\% of $\mathrm{Ar}$ atoms and $5 \%$ of $\mathrm{H}_{2}$ molecules) as a function of normalized time. The points are for CMDS, the solid lines (-) are for the BB model, the dashed lines (- - -) for the SC model and the dotted lines $(\cdots)$ for the HC model. The black, green, blue and red colors correspond to $g(\vec{v})=v$, $v^{2}, \vec{v} / v$ and $\vec{v}$, respectively. Note that in the first chart the red and blue dots are superimposed.

A further consistency of the $a b$ initio CMDS and the $\mathrm{BB}$ model can be clearly seen from the comparison of molecule speed, square of velocity, velocity orientation and velocity relaxation using the concept of autocorrela- 
tion functions introduced in Section III. The autocorrelation functions $\Phi_{g(\vec{v})}(t)$ for $g(\vec{v})=v, v^{2}, \vec{v} / v$ and $\vec{v}$ for pure $\mathrm{H}_{2}$ are shown in Fig. 3 (a). We used Eq. (15) for the BB model and Eq. (16) for CMDS, like in Refs. 51 and 58. For comparison, we also plotted in Fig. 3 (a) the autocorrelation functions for the phenomenological $\mathrm{HC}$ and SC models. For that, their effective frequency of velocity-changing collision was adjusted to the relaxation time of the velocity-autocorrelation function $\tau_{\vec{v}}$ obtained from CMDS. Analytical expressions for autocorrelation functions for $\mathrm{HC}$ and $\mathrm{SC}$ are given in Appendix B.

As can be seen in Fig. 3 (a), curves for CMDS, BB, and $\mathrm{HC}$ models are so close one to each other that they cannot be distinguished when one looks on each particular case of the quantity for which the autocorrelation function is calculated. It shows that if we compare only these four $\Phi_{v}, \Phi_{v^{2}}, \Phi_{\vec{v} / v}$, and $\Phi_{\vec{v}}$ autocorrelation functions then the CMDS, BB, and HC models are very much equivalent in the self-perturbing case. Far different results were obtained for the SC model. Apart from $\Phi_{\vec{v}}$, which was chosen such as to have the same relaxation time as CMDS, other autocorrelation functions, especially $\Phi_{v}$ and $\Phi_{v^{2}}$, for the SC model decay significantly faster than those for CMDS, BB, and HC models, what means that the analysis of these relaxation processes clearly distinguish the $\mathrm{SC}$ model from the three others.

To express the relaxation of the $g(\vec{v})$ functions quantitatively we determine the relaxation times of the autocorrelation functions from exponential fits to the curves from Fig. 3 (a) of the form $\Phi_{g(\vec{v})}(t)=C_{g(\vec{v})}^{(0)}+C_{g(\vec{v})}^{(1)} e^{-t / \tau_{g(\vec{v})}}$, see Table I. The fits for the $\mathrm{H}_{2}-\mathrm{H}_{2}$ system were performed for the time interval from 0 ps to $628 \mathrm{ps}$, while for the $\mathrm{H}_{2}$-Ar system, see Sec. VB, the interval was from 0 ps to 2500 ps. The constants $C_{g(\vec{v})}^{(0)}=\Phi_{g(\vec{v})}(\infty)=$ $\left[\int d^{3} \vec{v} f_{m}(\vec{v}) g(\vec{v})\right]^{2}$ and $C_{g(\vec{v})}^{(1)}+C_{g(\vec{v})}^{(0)}=\Phi_{g(\vec{v})}(0)=$ $\int d^{3} \vec{v} f_{m}(\vec{v})[g(\vec{v})]^{2}$ were determined from the autocorrelation functions behaviour for $t=0$, and $t \rightarrow \infty$. Their values for $\vec{v}, v^{2}, v$, and $\vec{v} / v$ are given in Ref. 51 . The agreement between results for CMDS and BB model demonstrates that the $a b$ initio $\mathrm{BB}$ model provides appropriate description of velocity-changing collisions for pure $\mathrm{H}_{2}$. Remind that the BB model does not make use of any adjustable parameters; the size of repulsive spheres was taken directly from the interaction potential and in this sense this model is labelled as ab initio.

At this point it should be noted that in general, when the collision operator $\hat{S}_{\mathrm{VC}}^{f}$ is not diagonal in the Burnett basis, $\nu_{\text {diff }}$ is not exactly equal to $1 / \tau_{\vec{v}}$ but only approximately. It is caused by the fact that in general the autocorrelation function $\Phi_{\vec{v}}$ cannot be represented by a simple decreasing exponential function, see Eqs. (14) and (15) and Refs. 60 and 61. Within the BB model, applied here for the $\mathrm{H}_{2}-\mathrm{H}_{2}$ system, the frequency of the velocity-changing collisions $\nu_{\text {diff }}$ is smaller than $1 / \tau_{\vec{v}}$ by about $0.7 \%$.

Table I contains also the relaxation times for $\mathrm{HC}$ and SC models. It was shown in Appendix B of Ref. 51
TABLE I. Relaxation times for CMDS and for the BB model (in ps). Results for $\mathrm{H}_{2}-\mathrm{H}_{2}$ for $n_{\mathrm{H}_{2}}=1 \mathrm{amg}$ and for $\mathrm{H}_{2}$-Ar for $n_{\mathrm{H}_{2}}=0.95 \mathrm{amg}$ and $n_{\mathrm{Ar}}=0.05 \mathrm{amg}$. The values of $\nu_{\mathrm{diff}}$ for the $\mathrm{HC}$ model and for the SC model were chosen such as to make their velocity relaxation times $\tau_{\vec{v}}$ equal to $\tau_{\vec{v}}$ obtained from CMDS. The wAr parameter is a percentage content of argon in $\mathrm{H}_{2}+$ Ar mixture.

\begin{tabular}{lrrrr}
\multicolumn{4}{c}{$\mathrm{H}_{2}-\mathrm{H}_{2}$} \\
\\
\hline \hline CMDS & $\tau_{\vec{v}}$ & $\tau_{v^{2}}$ & $\tau_{v}$ & $\tau_{\vec{v} / v}$ \\
$\mathrm{BB}$ & 113.4 & 122.7 & 124.6 & 113.8 \\
$\mathrm{HC}$ & 112.7 & 114.4 & 122.9 & 119.5 \\
$\mathrm{SC}$ & 113.4 & 113.4 & 113.4 & 113.4 \\
\hline \hline
\end{tabular}

\begin{tabular}{lrrrr}
\multicolumn{5}{c}{$\mathrm{H}_{2}-\mathrm{Ar}$} \\
\hline \hline & $\tau_{\vec{v}}$ & $\tau_{v^{2}}$ & $\tau_{v}$ & $\tau_{\vec{v} / v}$ \\
\hline $\mathrm{CMDS}_{\left(\mathrm{w}_{\mathrm{Ar}}=95 \%\right)}$ & 65.8 & 595.3 & 599.0 & 73.4 \\
$\mathrm{BB}_{\left(\mathrm{w}_{\mathrm{Ar}}=100 \%\right)}$ & 69.1 & 733.6 & 822.1 & 94.6 \\
$\mathrm{BB}_{\left(\mathrm{w}_{A r}=95 \%\right)}$ & 70.4 & 576.7 & 640.1 & 95.1 \\
$\mathrm{HC}$ & 65.8 & 65.8 & 65.8 & 65.8 \\
$\mathrm{SC}$ & 65.8 & 32.9 & 31.6 & 57.4 \\
\hline \hline
\end{tabular}

that within the $\mathrm{HC}$ model the relaxation times for any $g(\vec{v})$ functions are the same and equal to $1 / \nu_{\text {diff. }}$ The HC model is also called the single-relaxation time model. ${ }^{39}$ Comparing the very similar values of relaxation times for $v^{2}, v$, and $\vec{v} / v$ from the HC and CMDS models one may incorrectly interpret that the $\mathrm{HC}$ model gives a proper description of $\mathrm{H}_{2}-\mathrm{H}_{2}$ collisional kinetics. However, as we have already shown, by comparing the collision kernels (Fig. 2 (a)), the HC model gives a description of velocitychanging collisions for $\mathrm{H}_{2}-\mathrm{H}_{2}$, which is far from the $a b$ initio CMDS. These two models can be also clearly distinguished by comparing the relaxation times of higher powers of speed $v$, see Fig. 4 . For the HC model, the relaxation time $\tau_{v^{x}}$ is independent of $x$ as predicted in Ref. 51 but for CMDS we see a decrease of $\tau_{v^{x}}$ with an increase of $x$. One can also note that a similar, but not exactly the same, decrease is observed for the BB model. It also supports conclusion that the BB model reproduces much better results from CMDS than HC. The deviation between CMDS and the BB model is caused by the fact that the hard-sphere potential does not exactly reproduce the whole structure of the Lennard-Jones potential, in particular its minimum and attractive long-range part, see Fig. 1.

The relaxation times for the SC model were directly deduced from analytical formulas, Eqs. (B1-B4), given in Appendix B. Their values for the quantities $g(\vec{v})=v^{2}$ and $v$ are about two times smaller comparing to those from the three other approaches. It means that for the SC model the effective rate of speed-changing collisions $1 / \tau_{v}$ is two times larger than in the 3 other cases, namely CMDS, BB and HC models, see Refs. 27 and 58 and 

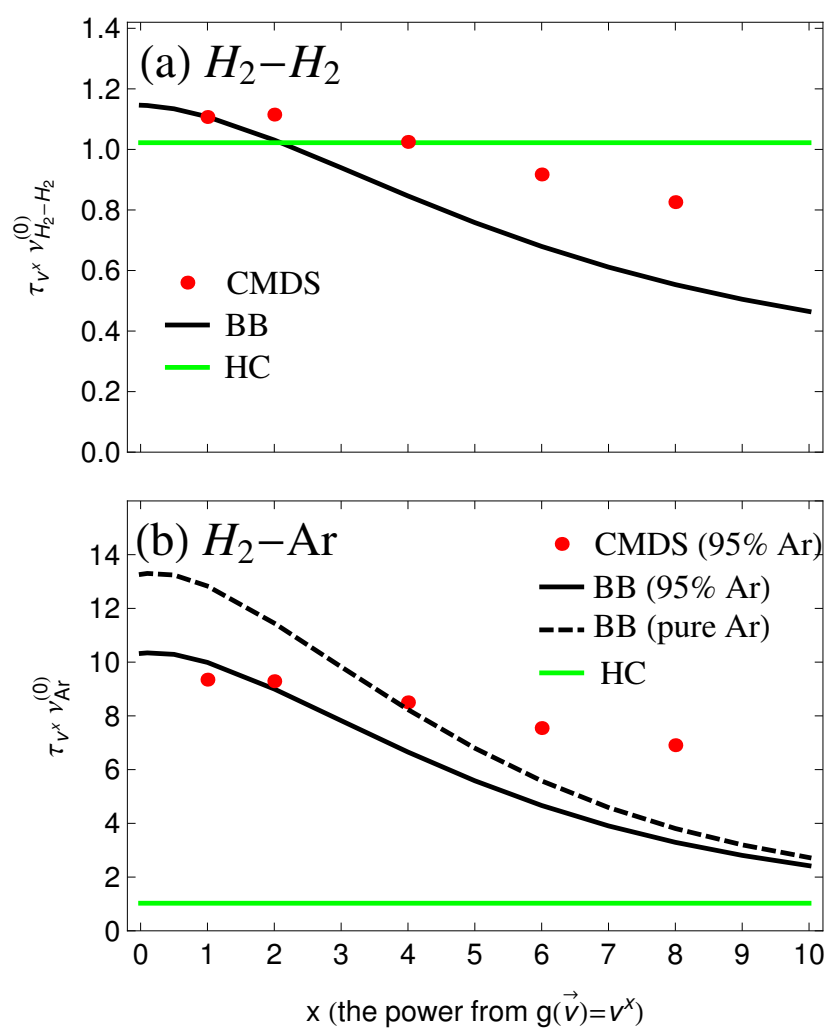

FIG. 4. (Color online) (a) Relaxation time $\tau_{v^{x}}$ of the quantity $g(\vec{v})=v^{x}$ as a function of power $x$ (the vertical axis is normalized by multiplication by the first-order effective frequency of velocity changing collision $\nu_{\mathrm{H}_{2}-\mathrm{H}_{2}}^{(0)}$ ) for pure $\mathrm{H}_{2}$. The red points correspond to the results obtained by CMDS while the black and green lines show the results for BB and $\mathrm{HC}$ models, respectively. (b) Relaxation time $\tau_{v^{x}}$ of the quantity $g(\vec{v})=v^{x}$ as a function of power $x$ for $\mathrm{H}_{2}$ diluted in Ar. Here the vertical axis is normalized by multiplication by $\nu_{H_{2}-A r}^{(0)}$.

\section{Appendix A.}

In this section we have demonstrated that, in contrast to the SC and $\mathrm{HC}$ models, the $a b$ initio $\mathrm{BB}$ model correctly reproduces the collisional kinetics of the $\mathrm{H}_{2}-\mathrm{H}_{2}$ system predicted by ab initio CMDS. ${ }^{51}$ From these results one can expect that the line profiles based on the BB model ${ }^{19,24}$ should be able to reproduce experimental spectra of self-broadened hydrogen more accurately than that based on the $\mathrm{HC}^{17,39,43,44}$ and $\mathrm{SC}^{17,24,38,42}$ models.

\section{B. $\mathbf{H}_{2}$-Ar collisions}

We analyze here $\mathrm{H}_{2}$-Ar collisions in very much similar way as it has been done for pure $\mathrm{H}_{2}$. Our main goal is to compare results from CMDS with those obtained by the $\mathrm{BB}, \mathrm{HC}$ and $\mathrm{SC}$ models in case of $5 \% \mathrm{H}_{2}+95 \%$ $\mathrm{Ar}$ mixture. Nevertheless, to better illustrate the role of small amount of $\mathrm{H}_{2}$ in this mixture, we also discuss properties of a mixture with $\mathrm{H}_{2}$ infinitesimally diluted in a bath of argon (the percentage content of argon in $\mathrm{H}_{2}$ + Ar mixture $\mathrm{w}_{A r} \rightarrow 100 \%$ ). In this section for all cases we assume that the total density number of the mixture is $n=n_{\mathrm{H}_{2}}+n_{\mathrm{Ar}}=1$ amg.

For the hard-sphere potential from Fig. 1 (c) and for $T=296 \mathrm{~K}$ the first-order effective frequency of velocity changing collision is $\nu_{\mathrm{H}_{2}-\mathrm{Ar}}^{(0)}=1 /(64.07 \mathrm{ps})$. Again, following Lindenfeld ${ }^{24,33}$ we found that for the ${ }^{40} \mathrm{Ar}$ to $\mathrm{H}_{2}$ mass ratio $\alpha=19.8^{59}$ the $f_{D}$ correction factor is 1.115 , what gives $\nu_{\text {diff, } \mathrm{H}_{2}-\mathrm{Ar}}=1 /(71.44 \mathrm{ps})$. The $\mathrm{BB}$ collision kernel, Eq. (5), for $\mathrm{H}_{2}$ perturbed by $\mathrm{Ar}$ is presented in Fig. 2 (c) and (d) as black, blue and red dashed lines. The corresponding relaxation times, evaluated also from $\mathrm{BB}$ model, are collected in the $\mathrm{BB}_{\left(\mathrm{w}_{\mathrm{Ar}}=100 \%\right)}$ row in Table I. With the $\mathrm{BB}$ model the value of $1 / \tau_{\vec{v}}$ is larger than the $\nu_{\text {diff }}$ frequency, as in the case of $\mathrm{H}_{2}-\mathrm{H}_{2}$ collisions. However here, for $\alpha \approx 20$, this difference is about $3.3 \%$, which is almost 5 times more than for pure $\mathrm{H}_{2}$ case, where the mass ratio is $\alpha=1$.

To relate the $\mathrm{BB}$ description of velocity-changing collisions to the CMDS reference we needed to consider a finite content of $\mathrm{H}_{2}$ in the $\mathrm{H}_{2}+\mathrm{Ar}$ mixture to achieve reasonably low numerical noise intrinsic to CMDS. We found that it is enough to take $5 \%$ of $\mathrm{H}_{2}$ and $95 \%$ of $\mathrm{Ar}$ ( $\mathrm{w}_{\mathrm{Ar}}=$ 0.95). For this mixture, the collision operator introduced in Eq. (2) can be expressed as the weighted mean $\hat{S}_{\mathrm{VC}, \text { mix }}=\left(1-\mathrm{w}_{\mathrm{Ar}}\right) \hat{S}_{\mathrm{VC}, \mathrm{H}_{2}-\mathrm{H}_{2}}+\mathrm{w}_{\mathrm{Ar}} \hat{S}_{\mathrm{VC}, \mathrm{H}_{2}-\mathrm{Ar}}$, where each of the operators $\hat{S}_{\mathrm{VC}, \mathrm{H}_{2}-\mathrm{H}_{2}}$ and $\hat{S}_{\mathrm{VC}, \mathrm{H}_{2}-\mathrm{Ar}}$ is given for $1 \mathrm{amg}$ number of density of pure $\mathrm{H}_{2}$ and Ar with infinitesimally diluted $\mathrm{H}_{2}$, respectively. Note, however, that the frequency of velocity-changing collisions $\nu_{\text {diff,mix }}$ corresponding to the $\hat{S}_{\mathrm{VC} \text {,mix }}$ operator , according to Appendix A, differs from a simple weighted mean $\left(1-\mathrm{w}_{\mathrm{Ar}}\right) \nu_{\mathrm{diff}, \mathrm{H}_{2}-\mathrm{H}_{2}}+\mathrm{w}_{\mathrm{Ar}} \nu_{\mathrm{diff}, \mathrm{H}_{2}}-\mathrm{Ar}$ by $2.4 \%$.

The collision kernels for $\mathrm{w}_{A r}=95 \%$ obtained from the CMDS and BB models are shown in Fig. 2 (c) and (d) as dots and solid lines, respectively. The BB model mostly correctly reproduces the CMDS results. Only for small $\theta_{k}$ angles these two approaches give different kernels (the magnitudes are different, while the shapes are similar), see the black solid lines and black dots in Fig. 2 (c) and (d). Underestimation of the magnitude of the scattering probability in this case by the BB model can be related to the lack of attractive interaction in this model. Corresponding relaxation times are collected in Table I. Relative differences between relaxation times obtained from BB and CMDS models are between $3.1 \%$ and $29.6 \%$ for $5 \%$ of $\mathrm{H}_{2}$ and $95 \%$ of Ar mixture, while for pure $\mathrm{H}_{2}$ these differences were between $0.6 \%$ and $6.8 \%$.

The reason why the $\mathrm{BB}$ model reproduces better the kinetics for the $\mathrm{H}_{2}-\mathrm{H}_{2}$ system than for the $\mathrm{H}_{2}$ - $\mathrm{Ar}$ case is that the mass ratio $\alpha$ is much higher for $\mathrm{H}_{2}$-Ar. As it was examined in Ref. 35, when the mass ratio $\alpha$ is higher then collisions dynamics are much more sensitive to the form of the potential, for a given effective frequency of velocity-changing collisions $\nu_{\text {diff }}$. Let us only remind that in the limiting case of $\alpha \rightarrow 0$ the collision 
operator $\hat{S}_{\mathrm{VC}}^{f}$ for any interaction potential becomes the Fokker-Planck operator, ${ }^{36}$ which does not depend on the interaction potential.

It is also purposeful to compare the $\mathrm{BB}$ results for $\mathrm{w}_{A r}=95 \%$ and $\mathrm{w}_{A r}=100 \%$. For the perturber bath consisting only of argon $\left(\mathrm{w}_{A r}=100 \%\right)$ the BB model relaxation time $\tau_{v^{2}}$ is by $20.7 \%$ longer than for mixture containing $5 \%$ of $\mathrm{H}_{2}$, see Table I. The presence of a small amount of the $\mathrm{H}_{2}$ perturber makes the speed relaxation significantly faster according to much smaller mass ratio $\alpha$, see Appendix A and Refs. 27 and 58. Similar difference is noticed for $\tau_{v}$. The other way of visualization of this effect is the comparison of the $\mathrm{BB}$ collision kernels for $\mathrm{w}_{A r}=95 \%$ and for $\mathrm{w}_{A r}=100 \%$, see Fig. 2 (c) and (d). In these figures the speed before collision $v_{i}$ is pointed by the thin vertical line. For speeds after collision $v_{j}$, which are far from $v_{i}$, the collision kernel for $\mathrm{w}_{A r}=100 \%$ is much smaller than that for $\mathrm{w}_{A r}=95 \%$, what means that it is much less probable to change the speed of an active molecule perturbed by pure argon. That explains the observed discrepancy of relaxation times related to the magnitude of speed or its square.

In Fig. 2 (c) and (d) we have also plotted the $\mathrm{HC}$ kernel as a gray dashed line setting the value of the effective frequency of velocity-changing collisions $\nu_{\text {diff }}$ to rate of velocity-changing collisions $1 / \tau_{\vec{v}}=1 /(65.8 \mathrm{ps})$ from CMDS. Despite the fact that in the $\mathrm{HC}$ model the

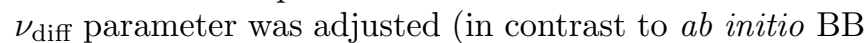
model, which originates only from potential parameters) the HC kernel reproduces much worse the CMDS collisional kernel than the BB kernel does. These discrepancies lead to huge differences in autocorrelation functions (see Fig. 3) and automatically to totally wrong values of relaxation times, see Table I. Similarly, the commonly used SC model is far from a proper description of velocity-changing collisions for the $\mathrm{H}_{2}$-Ar system. The effective rates of speed-changing collisions for the $\mathrm{HC}$ and $\mathrm{SC}$ models are about 10 and 20 times larger than that predicted by the BB model for $\mathrm{H}_{2}$-Ar collisions with $\alpha$ about $20 .{ }^{27,58}$

\section{APPLICATION TO PRECISE SPECTROSCOPIC MEASUREMENT}

In this section the BB model is used to analyze the experimental spectrum of the self-perturbed $\mathrm{H}_{2}$ first overtone $\mathrm{Q}(1)$ line recorded by high sensitivity Cavity Ring Down Spectroscopy (CRDS) near $8075 \mathrm{~cm}^{-1}$. Experimental conditions have been chosen in order to enhance the influence of velocity-changing collisions on the shape of the optical resonance, which allows to clearly distinguish the ab initio $\mathrm{BB}$ model from simple $\mathrm{HC}$ and $\mathrm{SC}$ models. The spectrum was recorded at a pressure of 640 Torr, which corresponds to the region of very high Dicke narrowing. In addition, the choice of the first overtone instead of a transition of the fundamental band leads to a broader Doppler effect, which enhances the impact of velocity-changing collisions on the optical resonance.

\section{A. Experimental details}

The spectrum of the $\mathrm{H}_{2} 2-0 \mathrm{Q}(1)$ line is displayed on Fig. 5 (a). It was measured in Grenoble with a newly developed CRDS spectrometer using a fibered External Cavity Diode Laser (ECDL: Toptica DL pro, $1200 \mathrm{~nm}$ ) as light source. The set up is very similar to the CRDS spectrometer based on Distributed Feed Back (DFB) which was used to achieve a record sensitivity of $5 \times 10^{-13} \mathrm{~cm}^{-1}$ for $\mathrm{CRDS}^{1}$ and only an outline of the data acquisition is given here. The $1.40 \mathrm{~m}$ long CRDS cell is fitted by very high reflectivity mirrors leading to ring down times of about $200 \mu \mathrm{s}$ in the considered spectral interval. The CRDS cell was filled with $\mathrm{H}_{2}$ (Fluka $99.999 \%$ stated purity) at 640 Torr. The pressure and the ring down cell temperature $(296.13 \mathrm{~K})$ were monitored during the spectrum acquisition. The number density $n=p /\left(k_{B} T\right)$ in this case is equal to 0.77676 amg. The absorption coefficient $\alpha_{\mathrm{abs}}(\omega)$ for given light frequency $\omega$ was obtained from the cavity ring down time

$$
\alpha_{\mathrm{abs}}(\omega)=\frac{1}{c \tau_{\mathrm{abs}}(\omega)}-\frac{1}{c \tau_{\mathrm{evac}}(\omega)},
$$

where $\tau_{\mathrm{abs}}(\omega)$ is the ring-down time of the cavity with absorbing gas, $\tau_{\text {evac }}(\omega)$ is the ring-down time of the evacuated cavity and $c$ is the light velocity.

The typical mode-hop free tuning range of this ECDL is about $0.8 \mathrm{~cm}^{-1}(24 \mathrm{GHz})$. The laser frequency was tuned over a $1.6 \mathrm{~cm}^{-1}(48 \mathrm{GHz})$ wide region around the line center by changing the grating angle together with the laser current. Three consecutive and partially overlapping spectra were recorded to cover the range of interest. About 10 ring down events were averaged for each spectral data point separated by $0.8 \times 10^{-3} \mathrm{~cm}^{-1}$ $(24 \mathrm{MHz})$. The noise equivalent absorption evaluated as the rms of the baseline fluctuation is $1 \times 10^{-11} \mathrm{~cm}^{-1}$. The absorption coefficient at line center being close to $1 \times 10^{-6} \mathrm{~cm}^{-1}$, the signal-to-noise ratio is about $10^{5}$. A further important advantage of this ECDL compared to DFB diode lasers used in Refs. 1 and 62 lies in its smaller linewidth, typically $0.003 \times 10^{-3} \mathrm{~cm}^{-1}(100 \mathrm{kHz}) \mathrm{com}-$ pared to $0.07 \times 10^{-3} \mathrm{~cm}^{-1}(2 \mathrm{MHz})$. Indeed, on the rising and falling portions of the profile, the DFB frequency jitter is inevitably converted to absorption fluctuations while no effect is visible in the feet and the top of the profile (see Fig. 1 of Ref. 62). Here, the frequency jitter is low enough to keep those intensity fluctuations below the intrinsic ring down noise level.

The wavenumber $\omega /(2 \pi c)$ of the light emitted by the laser diode was measured by a commercial Fizeau type wavemeter HighFinesse WSU7-IR, $0.17 \times 10^{-3} \mathrm{~cm}^{-1}$ $(5 \mathrm{MHz})$ resolution, $0.7 \times 10^{-3} \mathrm{~cm}^{-1}(20 \mathrm{MHz})$ accuracy over 10 hours, that allows laser frequency to be determined at a typical $100 \mathrm{~Hz}$ refresh rate. In order to further refine the absolute calibration, a very low pressure 
scan was recorded showing $\mathrm{H}_{2} \mathrm{O}$ lines (present as impurity) for which highly accurate positions are provided by the HITRAN database. ${ }^{63}$ The effective absolute accuracy of the laser wavenumber $\omega /(2 \pi c)$ was about $10^{-3} \mathrm{~cm}^{-1}$.

\section{B. Spectral analysis}

The shape of an isolated line affected by Doppler broadening, velocity-changing collisions leading to Dicke narrowing $^{7}$ as well as by speed-dependent collisional broadening and shifting ${ }^{16}$ can be calculated using theory based on solving of the proper transport/relaxation equation. ${ }^{19,21,53,64}$ We use here the formalism presented by Shapiro et al. ${ }^{53}$ In this formalism the spectral line shape is given by

$$
I(\omega)=\frac{1}{\pi} \operatorname{Re} \int d^{3} \vec{v} f_{m}(\vec{v}) h(\omega, \vec{v}),
$$

which is the real part of a Maxwellian averaged function $h(\omega, \vec{v})$. The function $h(\omega, \vec{v})$ is a solution of the following transport/relaxation equation ${ }^{19,53}$

$$
1=\left[-i\left(\omega-\omega_{0}-\vec{k} \cdot \vec{v}\right)-\hat{S}^{f}\right] h(\omega, \vec{v}),
$$

where $\omega-\omega_{0}$ is the light detuning, $\vec{k}$ is the wavevector of absorbed light and the operator $\hat{S}^{f}$ describes the influence of collisions on the shape of a spectral line. In order to numerically calculate the spectral line shape [Eq. (18)] the transport/relaxation equation [Eq. (19)] is solved converting it to the set of algebraic equations. It was done by decomposing operators and functions in the Burnett basis. ${ }^{19,24,25}$

For the purpose of this study, we assumed that correlation between phase-changing and velocity-changing collisions ${ }^{16,17,64-67}$ can be neglected. In this case, the collision operator can be written ${ }^{53}$

$$
\hat{S}^{f}=-\Gamma(v)-i \Delta(v)+\hat{S}_{\mathrm{VC}}^{f}
$$

in terms of the speed-dependent collisional width $\Gamma(v)=$ $\Gamma B_{W}\left(v / v_{m}\right)$ and shift $\Delta(v)=\Delta B_{S}\left(v / v_{m}\right)$ of the spectral line as well as the velocity-changing collision operator $\hat{S}_{\mathrm{VC}}^{f}$. Here $\Gamma=\int d^{3} \overrightarrow{\mathrm{v}} f_{m}(\overrightarrow{\mathrm{v}}) \Gamma(\mathrm{v})$ and $\Delta=$ $\int d^{3} \overrightarrow{\mathrm{v}} f_{m}(\overrightarrow{\mathrm{v}}) \Delta(\mathrm{v})$ are averaged collision width (HWHM half width at half maximum) and shift, respectively. The speed dependence of collisional broadening and shifting is described by the dimensionless functions $B_{W}(x)$ and $B_{S}(x)$, with $x=v / v_{m}$ the absorber reduced speed. ${ }^{8,68}$ Dimensionless functions $B_{W}(x)$ and $B_{S}(x)$ for $\mathrm{H}_{2} 2-0$ $\mathrm{Q}(1)$ line are not known. Therefore, like Tran et $a l,{ }^{51}$ we described the speed dependence of the broadening and shifting using an empirical relation explored for the $\mathrm{H}_{2}$ 1-0 band by Forsman et al. ${ }^{69}$ The $B_{S}(x)$ function was adjusted to properly reproduce magnitude of collisional shift $^{70,71}$ of the measured spectral line, see Appendix C for details. Note, however, that for molecular hydrogen the impact of collisional velocity changes on the molecular spectrum, at pressure investigated here, is significantly larger than the impact of collisional phase/state changes. Therefore, the use of empirical description of collisional phase/state change does not influence significantly the conclusions about the applicability of the $a b$ initio $\mathrm{BB}$ model of collisional velocity changes in $\mathrm{H}_{2}$ spectra. The problem of a detailed analysis of the speeddependent effects for the first overtone Q(1) line is out of the scope of this paper.

We used the BB model to describe the velocitychanging collisions, which allows us to test the considerations from Secs. II-V on experimental data. We call this line-shape profile the speed-dependent billiard-ball profile $\left(\mathrm{SD}_{e} \mathrm{BBP}\right),{ }^{24}$ where the $e$ subscript indicates that the empirical speed dependence of collisional broadening and shifting was used.

Because of the lack of precise knowledge from $a b$ initio calculations about speed-dependent collisional broadening and shifting, a direct comparison between the theoretical prediction and the measured line shape cannot be performed. The $\mathrm{SD}_{e} \mathrm{BBP}$ model was thus used to fit experimental data. The Doppler width $\omega_{D}=k v_{m}$ was set to the value corresponding to the gas temperature. Fitted parameters were the line area $A$, the effective frequency of the velocity-changing collisions $\nu_{\text {diff }}$, averaged width $\Gamma$, averaged shift $\Delta$ and unperturbed transition frequency $\omega_{0}$. The measured profile as well as the residuum from the fitted $\mathrm{SD}_{e} \mathrm{BBP}$ is presented in Fig. 5 (a) and (g), respectively. As can be observed in Fig. 5 (g), the residuum is dominated by the experimental noise mainly caused by the laser instability. The values of corresponding model parameters are collected in the penultimate row in Table II. Note that the accuracy of these numbers is much higher than experimental ones. We provide so many digits, because the scope of this section is not only to analyze the experimental spectrum, but also to estimate the systematic errors originating from a wrong choice of the line-shape model. Moreover this also demonstrates that the precision of the measured spectrum significantly exceeds its accuracy.

Figures 5 (b) - (e) show the residuals from NelkinGhatak profile (NGP), ${ }^{17,39}$ speed-dependent empirical Nelkin-Ghatak profile $\left(\mathrm{SD}_{e} \mathrm{NGP}\right),{ }^{43,44}$ Galatry profile $(\mathrm{GP})^{38}$ and the exact speed-dependent empirical Galatry profile $\left(\mathrm{SD}_{e} \mathrm{GP}\right),{ }^{24}$ respectively. Note that the NelkinGhatak and Galatry names indicate that the $\mathrm{HC}$ and $\mathrm{SC}$ models were used to describe the velocity-changing collisions, respectively. The exact $\mathrm{SD}_{e} \mathrm{GP}$ model ${ }^{24}$ is just $\mathrm{SD}_{e} \mathrm{BBP}$ with $\alpha=0$ and should not be mistaken with earlier versions called convoluted $\mathrm{SDGP}^{40}$ and approximated SDGP, ${ }^{42}$ see also discussion in Ref. 45 . It should be noted that the partially-Correlated Speed-Dependent Hard-Collision profile introduced by Pine, ${ }^{44}$ combined with quadratic speed-dependent collisional width and shift $^{72}$ (pCqSDHC), was recently recommended for construction of new spectroscopic databases and radiative transfer calculations. ${ }^{73}$ One of the reasons for which this 

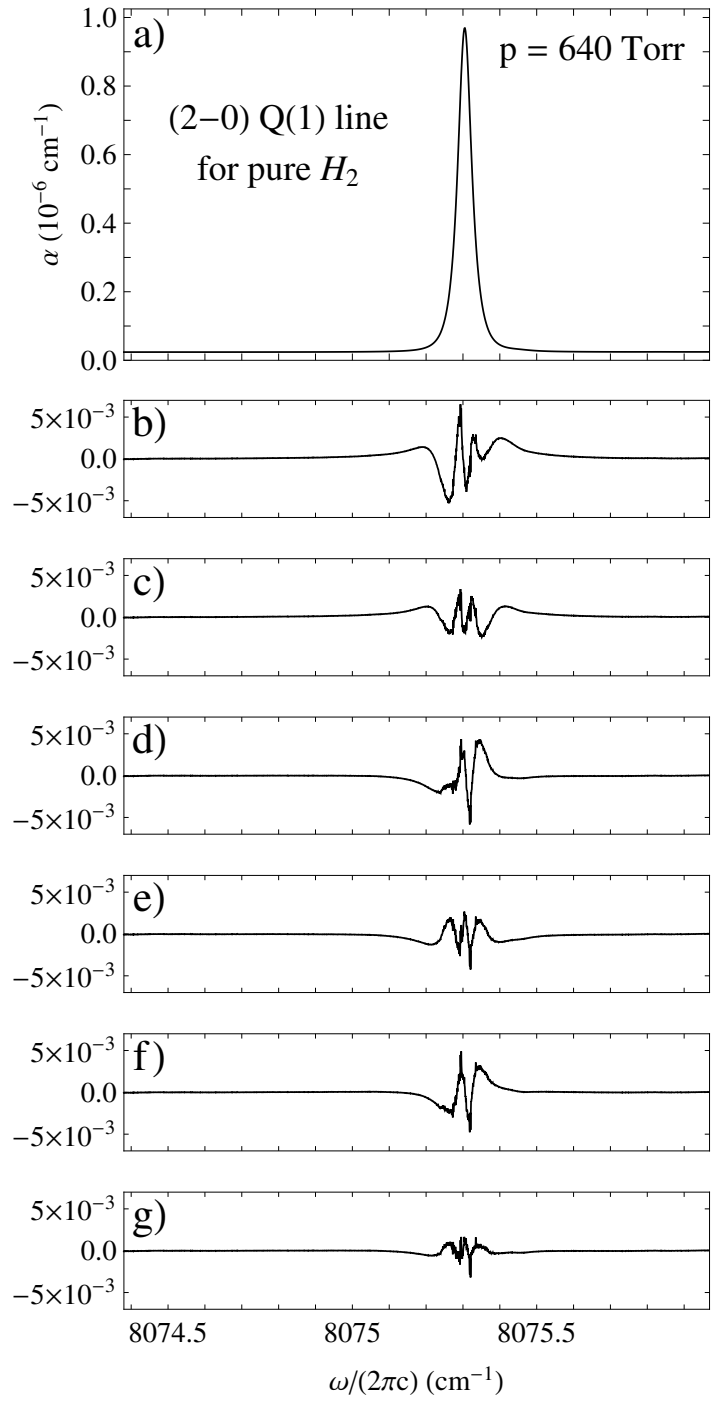

FIG. 5. (a) Experimental spectrum of self perturbed $\mathrm{H}_{2}$ first overtone $\mathrm{Q}(1)$ line measured at $p=640$ Torr and $T=296.13$ K. (b) - (g) Residuals from Nelkin-Ghatak profile (NGP), speed-dependent empirical Nelkin-Ghatak profile $\left(\mathrm{SD}_{e} \mathrm{NGP}\right)$, Galatry profile (GP), speed-dependent empirical Galatry profile ( $\left.\mathrm{SD}_{e} \mathrm{GP}\right)$, billiard-balls profile (BBP) and speed-dependent empirical billiard-balls profile $\left(\mathrm{SD}_{e} \mathrm{BBP}\right)$, respectively.

profile was chosen is that, in the case of quadratic speed dependence of collisional width and shift, ${ }^{72}$ a very efficient algorithm for its evaluation was proposed. ${ }^{74-77}$ While this profile allows for a very accurate description of the line shape of different molecular systems, for the particular case of the extremely light $\mathrm{H}_{2}$ molecule, it was shown that this pCqSDHC model leads to relatively large residuals, up to $3 \% .{ }^{74}$ More flexible description of spectral line shapes ${ }^{78}$ was proposed using a combination of soft and hard collisions ${ }^{9,11}$ and tested on $\mathrm{H}_{2}$ lines. $^{79,80}$

The values of fitted parameters are listed in Table II. In the cases of NGP, GP and BBP the collisional shift $\Delta$ has not been fitted, since for the models without speed dependency it is completely correlated to the $\omega_{0}$ parameter.

Comparing Fig. 5 (b) for NGP and Fig. 5 (c) for $\mathrm{SD}_{e} \mathrm{NGP}$ as well as Fig. 5 (d) for GP and Fig. 5 (e) for $\mathrm{SD}_{e} \mathrm{GP}$ one may observe that the presence of the speed dependence of collisional broadening and shifting reduces but does not eliminate the discrepancy with the experimental spectrum. These figures are in contrast with Fig. $5(\mathrm{~g})$ for the $\mathrm{SD}_{e} \mathrm{BBP}$. It should be noted that the same number of fitted parameters was used to fit the experimental spectrum in case of $\mathrm{SD}_{e} \mathrm{NGP}, \mathrm{SD}_{e} \mathrm{GP}$, and $\mathrm{SD}_{e} \mathrm{BBP}$.

For the $\mathrm{H}_{2}-\mathrm{H}_{2}$ system under the considered conditions, a proper description of velocity-changing collisions is crucial. When we compare magnitude of profiles parameters, listed in Table II, we clearly see that the line shape is dominated by the Doppler broadening affected by the velocity changing collisions. The speed-dependent collisional broadening and shifting are characterized by $\Gamma$ and $\Delta$, which are one order of magnitude smaller than $\omega_{D}$ describing Doppler broadening and $\nu_{\text {diff }}$ describing rate of the velocity-changing collisions. It is crucial to emphasise that the remaining residuals seen on Figs. 5 (c) and (e) are larger than those in Fig. 5 (g). It is a direct experimental manifestation of the improper description of velocity-changing collisions in pure $\mathrm{H}_{2}$ by the $\mathrm{HC}$ and SC models discussed in Sec. VA (see Figs. 2, 3 and 4). As it was already observed ${ }^{25,46,81}$ residuals of fitted line shapes at the Doppler regime are very sensitive to details of velocity-changing collisions. The remaining residuals from $\mathrm{SD}_{e} \mathrm{BBP}$, Fig. $5(\mathrm{~g})$, can be caused by the use of a simple phenomenological width and shift speed dependence. The other source of differences can be due to the fact that the billiard-ball model does not perfectly reproduce the $\mathrm{H}_{2}-\mathrm{H}_{2}$ potential. It was shown in Ref. 35 how the change of the interaction potential can affect the Dicke narrowed spectrum.

Since the $\mathrm{SD}_{e} \mathrm{BBP}$ leads to the best description of the shape of measured spectral line, it is worthy to analyze in more detail the results obtained with this model. The frequency of the velocity-changing collisions per molecule density, $\nu_{\text {diff }} / n$, determined from the $\mathrm{SD}_{e} \mathrm{BBP}$ fit was $1 /(125.29) \mathrm{ps}^{-1} \mathrm{amg}^{-1}$, while we obtained, at $296 \mathrm{~K}$, $1 /(113.45) \mathrm{ps}^{-1} \mathrm{amg}^{-1}$ with our BB model (Sec. V A). As can be seen the fitted one is smaller by almost $12 \%$. Note that if the frequency of the velocity-changing collisions is known for a given pressure $p_{1}$ and temperature $T_{1}$ then, within the BB model, it can be easily recalculated for other conditions characterised by some other $p_{2}$ and $T_{2}$ via the relation $\nu_{\text {diff }}\left(p_{1}, T_{1}\right) / \nu_{\text {diff }}\left(p_{2}, T_{2}\right)=$ $\left(p_{1} / p_{2}\right) \sqrt{T_{2} / T_{1}}$. Furthermore, the frequency of velocitychanging collisions can be related to the diffusion coefficient $D$ from Eq. (A1) and compared with other experimental results. Diffusion coefficients obtained by us for number density $1 \mathrm{amg}$ and temperature $296 \mathrm{~K}$ are 
TABLE II. Model parameters retrieved from the fits to the experimental spectrum of the 2-0 Q(1) line of $\mathrm{H}_{2}$ near $8075 \mathrm{~cm}^{-1}$. The Doppler broadening was fixed to $\omega_{D} /(2 \pi c)=0.0420997 \mathrm{~cm}^{-1}$, since the measurement was performed at $T=296.13 \mathrm{~K}$. The spectrum was recorded at a pressure of 640 Torr, what within the ab initio calculations for the BB model leads to $\nu_{\text {diff }} /(2 \pi c)=0.0364 \mathrm{~cm}^{-1}$.

\begin{tabular}{clllll}
\hline \hline Line profile & $A\left(10^{-6} \mathrm{~cm}^{-2}\right)$ & $\frac{\nu_{\text {diff }}}{2 \pi c}\left(\mathrm{~cm}^{-1}\right)$ & $\frac{\Gamma}{2 \pi c}\left(\mathrm{~cm}^{-1}\right)$ & $\frac{\Delta}{2 \pi c}\left(\mathrm{~cm}^{-1}\right)$ & $\frac{\omega_{0}}{2 \pi c}\left(\mathrm{~cm}^{-1}\right)$ \\
\hline $\mathrm{NGP}$ & $0.0537103(195)$ & $0.029260(76)$ & $0.0028751(228)$ & $-0.002874($ fixed $)$ & $8075.307776(5)$ \\
$\mathrm{SD}_{e} \mathrm{NGP}$ & $0.0538637(111)$ & $0.026065(27)$ & $0.0032163(136)$ & $-0.003182(17)$ & $8075.308470(24)$ \\
$\mathrm{GP}$ & $0.0542928(140)$ & $0.042220(60)$ & $0.0042908(153)$ & $-0.002874($ fixed $)$ & $8075.307775(3)$ \\
$\mathrm{SD}_{e} \mathrm{GP}$ & $0.0544043(90)$ & $0.036999(28)$ & $0.0045487(102)$ & $-0.003455(17)$ & $8075.308695(22)$ \\
$\mathrm{BBP}$ & $0.0541343(128)$ & $0.038174(55)$ & $0.0039175(144)$ & $-0.002874($ fixed $)$ & $8075.307776(3)$ \\
$\mathrm{SD}_{e} \mathrm{BBP}$ & $0.0542404(46)$ & $0.032920(13)$ & $0.0041715(53)$ & $-0.002874(7)$ & $8075.308114(10)$ \\
$\mathrm{SD}_{e} \mathrm{BBP}$ & $0.0542404(45)$ & $0.032920(13)$ & $0.0041715(53)$ & $-0.002874($ fixed $)$ & $8075.308114(1)$ \\
\hline \hline
\end{tabular}

$1.530 \mathrm{~cm}^{2} / s$ for $\mathrm{SD}_{e} \mathrm{BBP}$ fit and $1.385 \mathrm{~cm}^{2} / \mathrm{s}$ for $a b i n i$ tio $\mathrm{BB}$ model. The earlier experimental determinations, corresponding also to $1 \mathrm{amg}$ and $296 \mathrm{~K}$, are $1.35 \mathrm{~cm}^{2} / \mathrm{s}^{82}$ and $1.34 \mathrm{~cm}^{2} / \mathrm{s}^{83}$

The $12 \%$ underestimation of the $\nu_{\text {diff }}$ frequency obtained from $\mathrm{SD}_{e} \mathrm{BBP}$ fit suggests that for this system the dephasing and velocity-changing contributions are slightly correlated. Moreover the simplified empirical treatment of the collisional broadening and shifting speed dependence can also contribute to these differences. For instance, we verified that the use of hypergeometric speed dependence of $\Gamma(v)$ and $\Delta(v)$ leads to $14 \%$ change of $\nu_{\text {diff. }}$ Nevertheless, to be sure about it, a full quantum calculations of $\mathrm{H}_{2}-\mathrm{H}_{2}$ scattering taking into account both the dephasing and velocity-changing parts should be performed.

It is easy to see from Table II, that the use of improper description of velocity-changing collisions can lead to significant systematic error of the retrieved line-shape parameters. It is especially important if the aim is ultraprecise determination of some line parameters. To show the potential of the data used here, we fixed $\Delta$ in our fit to remove the strong correlation between $\Delta$ and $\omega_{0}$. In fact, such correlation can be removed recording various spectra at different pressure and using a multispectrum fitting technique. ${ }^{84}$ This may allow a potential determination of the line position, as whole including $\Delta$ and $\omega_{0}$, with a precision of about $10^{-6} \mathrm{~cm}^{-1}(30 \mathrm{kHz})$. At this level of precision a proper description of collisions is crucial for an accurate determination of line frequency.

Taking into account that an effective absolute accuracy of the laser frequency was about $10^{-3} \mathrm{~cm}^{-1}$, the unperturbed frequency of the first overtone $\mathrm{Q}(1)$ line determined from $\mathrm{SD}_{e} \mathrm{BBP}$, see Table II, is consistent with the previous theoretical value $8075.3074 \mathrm{~cm}^{-1}$ reported in Ref. 49 based on the approach developed by Komasa et $a l .{ }^{85}$ On the other hand the thermally averaged collisional shift can be estimated from the single line shape fitting thanks to its speed dependence, however, result will be dramatically dependent on the form of assumed $B_{S}(x)$ function. Finally, the line intensity determined from $\mathrm{SD}_{e} \mathrm{BBP} 25.990 \times 10^{-28} \mathrm{~cm}^{-1} /\left(\right.$ molecule $\left./ \mathrm{cm}^{2}\right)$ agrees very well, within $0.04 \%$, with the theoretical value $25.980 \times 10^{-28} \mathrm{~cm}^{-1} /\left(\right.$ molecule $\left./ \mathrm{cm}^{2}\right)$ reported in Ref. 49 . Note that the use of simplified models leads to significantly higher discrepancies with this theoretical value. For instance for speed-dependent line shapes the discrepancies are $-0.66 \%$ and $0.34 \%$ for $\mathrm{SD}_{e} \mathrm{NGP}$ and $\mathrm{SD}_{e} \mathrm{GP}$, respectively, while for speed-independent line shapes the discrepancies are $-0.94 \%$ and $0.13 \%$ for NGP and GP, respectively.

The other parameters of the line shape will not be discussed in so much detail. The magnitude of $\Gamma$ or $\Delta$ (and consequently also $\omega_{0}$ ) will very strongly depend on assumed or fitted speed dependence of collisional broadening and shifting. It would also require analysis of measured line shapes in a large range of pressures, but again this is out of the scope of this paper.

Handling the physical mechanisms underlying the line shape models is of huge importance for metrological applications, because it allows not only to obtain the model fit with smaller residuum, but also to reduce the systematic errors of retrieved parameters in a controlled way. Clearly, the use of realistic description of collisions in line shape calculations is crucial for different applications such as the spectroscopic determination of the Boltzmann constant, $4,5,46,86$ testing quantum treatment of molecules with high precision (in particular, line intensities) ${ }^{49}$ or search for new fundamental physical effects. ${ }^{87,88}$

Comparing the descriptions of the velocity-changing collisions given by various models (see Sec. VA) and corresponding residua (see Fig. 5) it is clear that from these approaches the BB model gives the best description of velocity-changing collisions for pure $\mathrm{H}_{2}$. For further development several improvements will be required. The description of the velocity-changing collisions should be based on realistic interaction potential having a long range attractive part instead of the simple hard-sphere potential. The empirical speed dependence of collisional broadening and shifting should be replaced with results obtained from $a b$ initio quantum-mechanical calculations of $\mathrm{H}_{2}-\mathrm{H}_{2}$ scattering performed for the appropriate first overtone line. Ultimately, the velocity changing and dephasing should be coherently included in the 
collision operator determining the shape of the spectral line. This fully $a b$ initio line shape calculation based on quantum-mechanical scattering of $\mathrm{H}_{2}$, intrinsically, should also resolve how important the correlations between the velocity-changing and dephasing contributions are.

It was demonstrated that $\mathrm{H}_{2}$ rovibrational transitions are very sensitive to speed dependence of collisional broadening and shifting as well as to details of velocitychanging collisions. Moreover, because of its simplicity, $\mathrm{H}_{2}$ can be described accurately in the ab initio way. Therefore it is an excellent system for testing line shape models. In molecular systems containing $\mathrm{H}$ very often deviations from Voigt profile are enhanced comparing to other atmospheric molecules. Critical verification of line shape models in this kind of systems can be very helpful for construction of new spectroscopic data basis. ${ }^{73,78,89}$ Especially this is important for minimizing and quantifying the systemic errors of collected data. Line shape models verified on more demanding systems can be applied to other molecular systems, important in atmospheric research, which are less sensitive to speed dependences of collisional broadening or velocity-changing collisions.

\section{SUMMARY}

In this paper we quantified, in $a b$ initio way, the systematic errors in $\mathrm{H}_{2}$ spectra analysis caused by the use of oversimplified models of the velocity-changing collisions like the soft- or hard-collisions models, which are commonly applied in molecular spectra interpretation. These results are crucial from the point of view of recent very rapid developments of ultra-accurate spectroscopic measurements, where effective accuracy is no longer limited by experimental imperfections, but by the use of wrong line-shape models. ${ }^{4,5,25}$

We showed that the BB model properly describes the velocity-changing collisions for $\mathrm{H}_{2}-\mathrm{H}_{2}$ and $\mathrm{H}_{2}$ - $\mathrm{Ar}$. We did it by relating the results obtained from the $\mathrm{BB}$ model to those from $a b$ initio CMDS. Firstly, we showed a consistency between very principal collisional properties like frequency of the velocity-changing collisions or the collision kernels. Then we developed an operator formalism, which allows to evaluate the autocorrelation functions for any model of the velocity-changing collisions. That allowed us to show that the gas kinetics, characterized by autocorrelation functions relaxations, predicted by the $\mathrm{BB}$ model is consistent with those obtained from ab initio CMDS. Moreover, we showed that these collisional properties derived from the simple phenomenological soft- and hard-collision models are far different than those resulted from the BB model and CMDS.

The accurate description of collisional velocity changes provided by the BB model is know well supported by its good agreement with CMDS and experimental spectra. Application of this model seems to be essential to improve effective accuracy of optical metrology of molecular sys- tems based on the Doppler-limited spectroscopy. It is especially important for molecular hydrogen at pressures well below the high-pressure regime, where the collisional effects related to the velocity changes have significantly bigger impact on the spectral line shape than the effects related to molecule phase and state changes. For other systems, well controlled quantification of these systematic errors will allow to increase the accuracy in many, recently rapidly developing, fields of optical metrology like spectroscopic determination of Boltzmann constant, where at the moment the main contribution to the error budget comes from oversimplified treatment of the spectral line shape. ${ }^{4,5}$

\section{ACKNOWLEDGEMENTS}

The authors would like to thank J.-M. Hartmann for stimulating discussions. The research is a part of the program of the National Laboratory FAMO in Torun, Poland. The research is partially supported by the Foundation for Polish Science TEAM Project co-financed by the EU European Regional Development Fund, and is partially supported by the National Science Centre, Poland, Project No. DEC-2013/09/N/ST4/00327 and DEC-2011/01/B/ST2/00491. The financial support provided by the PHC POLONIUM program is acknowledged. RC would like to thank the University of Paris Est Créteil for invitation as guest researcher in 2013. HT thanks the Institut du Développement et des Ressources en Informatique Scientifique (IDRIS) for giving access to the IBM Blue Gene/Q parallel computer for the CMDS calculations. The authors at LIPhy are part of Labex OSUG@2020 (ANR10 LABX56).

\section{Appendix A: Effective frequency of velocity-changing collisions}

The effective frequency of velocity-changing collisions

$$
\nu_{\text {diff }}=\frac{v_{m}^{2}}{2 D}
$$

related to the diffusion coefficient $D$ can be evaluated directly from the velocity-changing collisions operator $\hat{S}_{\mathrm{VC}}^{f}$. When a given operator $\hat{S}_{\mathrm{VC}}^{f}$ is decomposed in the Burnett functions basis then a corresponding effective frequency of velocity-changing collisions $\nu_{\text {diff }}$ can be found solving the set of following linear equations for $a_{n}$ coefficients $^{24,33}$

$$
-\sum_{n^{\prime}=0}^{\infty}\left[\hat{S}_{\mathrm{VC}}^{f}\right]_{n 1, n^{\prime} 1} a_{n^{\prime}}=\delta_{n, 0}
$$

where $\left[\hat{S}_{\mathrm{VC}}^{f}\right]_{n l, n^{\prime} l^{\prime}}$ are matrix elements of the $\hat{S}_{\mathrm{VC}}^{f}$ operator in the Burnett basis. Here we use the notation from Ref. 24. Note that the Burnett functions are identified with two numbers $l$ and $n,{ }^{54}$ however in Eq. (A2) only the 
elements with $l=1$ are needed. The effective frequency of the velocity-changing collisions is given by $\nu_{\text {diff }}=1 / a_{0}$, where $a_{0}$ is solution of Eq. (A2).

The first-order approximation of $\nu_{\text {diff }}$, which also approximates $\tau_{\vec{v}}$, is $\nu^{(0)}=-\left[\hat{S}_{\mathrm{VC}}^{f}\right]_{01,01} \approx 1 / \tau_{\vec{v}}$. The ratio of $\nu^{(0)}$ and $\nu_{\text {diff }}$ is denoted as $f_{D}=\nu^{(0)} / \nu_{\text {diff. }}$ The firstorder approximation can provide also information about speed-changing collisions. The effective rate of speedchanging collisions can be characterized by $\tau_{v^{2}}$ the relaxation time of $v^{2}$. The first-order effective rate of speedchanging collisions can be given in the following form $1 / \tau_{v^{2}} \approx-\left[\hat{S}_{\mathrm{VC}}^{f}\right]_{10,10}$. Both these expressions given in the first-order approximation for the effective frequency of velocity-changing collisions as well as for the effective rate of speed-changing collisions are exact if the collision operator $\hat{S}_{\mathrm{VC}}^{f}$ is diagonal in the Burnett basis. This is the case for the SC or HC models. Within the framework of the BB model, expression for the first-order approximation to the effective rate of speed-changing collisions can be written as $1 / \tau_{v^{2}} \approx \nu^{(0)} 2 /(1+\alpha)$, see Ref. 27 .

In case of gas mixture the velocity-changing collision operator $\hat{S}_{\mathrm{VC}, \text { mix }}$ can be written as sum

$$
\hat{S}_{\mathrm{VC}, \operatorname{mix}}=\sum_{i} \hat{S}_{\mathrm{VC}, i}
$$

of operators $\hat{S}_{\mathrm{VC}, i}$ describing velocity-changing collisions between the molecule under consideration and each component of the mixture. It is obvious that the first-order effective frequencies of velocity-changing collisions $\nu_{\text {mix }}^{(0)}$ and $\nu_{i}^{(0)}$ corresponding to operators $\hat{S}_{\mathrm{VC}, \text { mix }}$ and $\hat{S}_{\mathrm{VC}, i}$, respectively, fulfil ordinary sum relation $\nu_{\text {mix }}^{(0)}=\sum_{i} \nu_{i}^{(0)}$. The same can be shown for effective rates of speedchanging collisions in the first-order approximation. The ordinary sum will be also exact for not approximated quantities if all collision operators $\hat{S}_{\mathrm{VC}, i}^{f}$ are diagonal in the Burnett basis, however it is not true in a general case.

Possible discrepancies between $\nu_{\text {diff,mix }}$ and ordinary $\operatorname{sum} \sum_{i} \nu_{\mathrm{diff}, i}$ can be illustrated on example of two component gas mixture. We consider two components of perturbers 1 and 2. Velocity-changing collisions of active molecules are described by BB model with perturber to active molecule mass ratios $\alpha_{i}$ and effective frequency of velocity-changing collisions $\nu_{\mathrm{diff}, i}$ given for each component. We will focus on the case were $\alpha_{1}=1$, typical in mixture were some self-broadening takes place and the other $\alpha_{2}$ varies. In Figure 6 for different $\alpha_{2}$ we present dependence of the ratio $y=\nu_{\text {diff,mix }} /\left(\nu_{\text {diff, }, 1}+\nu_{\text {diff, }, 2}\right)$ on the relative contribution of component 1 to all collisions caused by both components 1 and $2, x=\nu_{\text {diff, } 1} /\left(\nu_{\text {diff, } 1}+\right.$ $\left.\nu_{\text {diff,2 }}\right)$. If only collisions caused by component 1 are important $x=1$. In the opposite case, where only collisions caused by component 2 are important $x=0$. In a middle case for $0<x<1$ both components 1 and 2 are contributing to velocity changing collisions.

As can be seen from Fig. 6, if components have very different masses, like it is in the case of $\mathrm{H}_{2}-\mathrm{Ar}$,

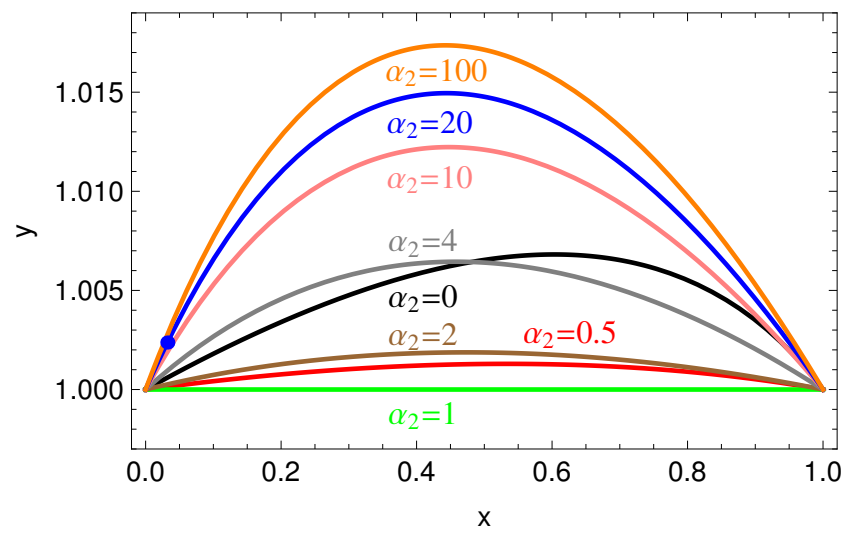

FIG. 6. (Color online) Relation between a normalized effective frequency of the velocity-changing collisions for a two component mixture, $y=\nu_{\text {diff,mix }} /\left(\nu_{\text {diff, } 1}+\nu_{\text {diff, } 2}\right)$ and the parameter $x=\nu_{\text {diff, } 1} /\left(\nu_{\text {diff, } 1}+\nu_{\text {diff, } 2}\right)$. This result was obtained with the BB model. The first component of the perturber was assumed to have the same mass as the considered molecule $\left(\alpha_{1}=1\right)$, while the mass ratio of the second component of the perturber to the considered molecule was varying from $\alpha_{2}=0$ up to 100 .

$\nu_{\text {diff,mix }}$ can be larger at most by $1.5 \%$ than ordinary sum $\nu_{\text {diff, } \mathrm{H}_{2}-\mathrm{H}_{2}}+\nu_{\text {diff, } \mathrm{H}_{2}-\mathrm{Ar}}$. In a more common case of atmospheric gases where their mass differ not more than by factor of two, the discrepancies will be below $0.2 \%$. For the mixture of $5 \% \mathrm{H}_{2}+95 \%$ Ar, from ab initio $\mathrm{BB}$ model we have $\nu_{\text {diff, } \mathrm{H}_{2}-\mathrm{H}_{2}}=0.05 /(113.45 \mathrm{ps})=0.44 \times$ $10^{9}(1 / s)$ in 0.05 amg and $\nu_{\text {diff, } \mathrm{H}_{2}-\mathrm{Ar}}=0.95 /(71.44 \mathrm{ps})=$ $13.30 \times 10^{9}(1 / s)$ in $0.95 \mathrm{amg}$ what gives $x=0.032$ and $y=1.0024$ marked as a blue dot in Fig. 6. As can be seen the correction to the ordinary sum of the effective frequencies of velocity-changing collisions is less than $0.3 \%$.

In case of the effective rates of speed-changing collisions, also from ab initio $\mathrm{BB}$ model, we have $1 / \tau_{v^{2} \text {, mix }}=$ $1 /(576.7 \mathrm{ps})$ in $1 \mathrm{amg}, 1 / \tau_{v^{2}, \mathrm{H}_{2}}=0.05 /(114.4 \mathrm{ps})$ in 0.05 amg and $1 / \tau_{v^{2}, \mathrm{Ar}}=0.95 /(733.6 \mathrm{ps})$ in $0.95 \mathrm{amg}$ what gives $x=0.252$ and $y=1.0012$. The correction to the ordinary sum of the effective rates of speed-changing collisions is $0.12 \%$.

Discussion presented in this appendix is also related to earlier work by Hoang et al, ${ }^{58}$ where properties of the relaxation times ratio $\tau_{\vec{v}} / \tau_{v}$, called therein the memory parameter, for mixtures were investigated. They showed nonlinearity of the $\tau_{\vec{v}} / \tau_{v}$ ratio with fractional concentration of the mixture.

On the other hand the ratio of matrix elements $\left[\hat{S}_{\mathrm{VC}}^{f}\right]_{10,10} /\left[\hat{S}_{\mathrm{VC}}^{f}\right]_{01,01}$, which in first order approximates the relaxation times ratio $\tau_{\vec{v}} / \tau_{v^{2}}$, was introduced in Ref. 27 as an estimation of so called hardness parameter. ${ }^{27} \mathrm{~A}$ very good consistency between results from Refs. 27 and 58, for a wide range of perturber to absorber mass ratio $\alpha$, was demonstrated. 


\section{Appendix B: Autocorrelation functions for hard and soft collisions}

In the limit of $\alpha \rightarrow 0$ the BB model as well as any other model strictly based on interaction potential of colliding molecules is equivalent to the SC model. In this case the autocorrelation functions given by Eq. (15) for $g(\vec{v})=\vec{v}$, $v^{2}, v$ and $\vec{v} / v$ take simple analytical forms

$$
\begin{gathered}
\Phi_{\vec{v}}(t)=v_{m}^{2} \frac{3}{2} e^{-\nu_{\text {diff }} t}, \\
\Phi_{v^{2}}(t)=v_{m}^{4}\left(\frac{9}{4}+\frac{3}{2} e^{-2 \nu_{\text {diff }} t}\right), \\
\Phi_{v}(t)=v_{m}^{2} \sum_{n=0}^{\infty} \frac{(1+2 n)(2 n) !}{\pi\left(4 n^{2}-1\right)^{2}(n !)^{2} 4^{n-1}} e^{-2 n \nu_{\text {diff }} t}, \\
\Phi_{\vec{v} / v}(t)=\sum_{n=0}^{\infty} \frac{2(2 n) !}{\pi\left(\frac{3}{2}+n\right)\left(\frac{1}{2}+n\right)(n !)^{2} 4^{n}} e^{-(1+2 n) \nu_{\text {diff }} t},
\end{gathered}
$$

where $\nu_{\text {diff }}$ is an effective frequency of velocity-changing collisions. Equations (B1) and (B2) are equal to the formulas derived with the Keilson-Storer model in the softcollisions limit presented in Ref. 51. In contrast to the $\mathrm{SC}$ model, all autocorrelation functions for the $\mathrm{HC}$ model decay with the same rate ${ }^{51}$

$$
\begin{gathered}
\Phi_{\vec{v}}(t)=v_{m}^{2} \frac{3}{2} e^{-\nu_{\mathrm{diff}} t} \\
\Phi_{v^{2}}(t)=v_{m}^{4}\left(\frac{9}{4}+\frac{3}{2} e^{-\nu_{\mathrm{diff}} t}\right), \\
\Phi_{v}(t)=v_{m}^{2}\left[\frac{4}{\pi}+\left(\frac{3}{2}-\frac{4}{\pi}\right) e^{-\nu_{\mathrm{diff}} t}\right], \\
\Phi_{\vec{v} / v}(t)=e^{-\nu_{\mathrm{diff}} t} .
\end{gathered}
$$

\section{Appendix C: Empirical speed dependence of collisional broadening and shifting}

Following Forsman et $a l^{69}$ and Tran et $a l,{ }^{51}$ we assumed that the dependence of the collisional width $\Gamma^{(r)}\left(v_{r}\right)$ and shift $\Gamma^{(r)}\left(v_{r}\right)$ on the relative perturberabsorber speed $v_{r}$ can be approximated by simple analytical functions:

$$
\begin{aligned}
& \Gamma^{(r)}\left(v_{r}\right)=\mathcal{A}+\mathcal{B} v_{r}^{2}, \\
& \Delta^{(r)}\left(v_{r}\right)=\mathcal{C}+\mathcal{D} v_{r},
\end{aligned}
$$

where $\mathcal{A}, \mathcal{B}, \mathcal{C}$ and $\mathcal{D}$ are empirical constants. The speeddependent collisional width $\Gamma(v)$ and shift $\Delta(v)$ as a functions of the absorber speed $v$ :

$$
\Gamma(v)=\left(\mathcal{A}+\frac{3}{2} v_{m}^{2} \mathcal{B}\right)+\mathcal{B} v^{2},
$$

$$
\begin{aligned}
& \Delta(v)= \\
& \mathcal{C}+v_{m} \mathcal{D}\left(\frac{1}{\sqrt{\pi}} e^{-\left(v / v_{m}\right)^{2}}+\frac{1}{2}\left(1+2\left(v / v_{m}\right)^{2}\right) \frac{\operatorname{Erf}\left(v / v_{m}\right)}{\left(v / v_{m}\right)}\right)
\end{aligned}
$$

are obtained by averaging Eqs. (C1) and (C2) over Maxwellian distribution of perturber velocities, see Ref. 68. The averaged collision width $\Gamma$ and shift $\Delta$ are given by the following expressions:

$$
\begin{gathered}
\Gamma=\mathcal{A}+3 \mathcal{B} v_{m}^{2}=\mathcal{A}+\left(\mathcal{B} \frac{6 k_{B}}{m_{1}}\right) T \\
\Delta=\mathcal{C}+\frac{2 \sqrt{2}}{\sqrt{\pi}} \mathcal{D} v_{m}=\mathcal{C}+\left(\mathcal{D} \sqrt{\frac{16 k_{B}}{\pi m_{1}}}\right) \sqrt{T} .
\end{gathered}
$$

Note that Equations (C3-C6) are valid only for the selfperturbed case $(\alpha=1)$.

The speed dependence of collisional broadening and shifting is described by the dimensionless functions $B_{W}(x)$ and $B_{S}(x)$ defined as: 8,68

$$
B_{W}(x)=\frac{\Gamma\left(x v_{m}\right)}{\Gamma},
$$

and

$$
B_{S}(x)=\frac{\Delta\left(x v_{m}\right)}{\Delta} .
$$

The speed dependencies of collision broadening and shifting for $\mathrm{H}_{2}$ 2-0 Q(1) line are not known. Therefore, like Tran et al, ${ }^{51}$ we approximate $B_{W}(x)$ and $B_{S}(x)$ functions by functions corresponding to $\mathrm{H}_{2}$ 1-0 $\mathrm{Q}(1)$ transition, deduced from experimental data reported by Forsman et al. ${ }^{69}$ Following Forsman et $a l,{ }^{69}$ we took $\mathcal{A}=-6.1 \times 10^{-4} \mathrm{~cm}^{-1}, \mathcal{B}=2.01 \times 10^{-10} \mathrm{~cm}^{-1} /\left(\mathrm{m}^{2} / \mathrm{s}^{2}\right)$ and $\mathcal{C}=-0.017 \mathrm{~cm}^{-1}$. We adjusted $\mathcal{D}=4.39 \times$ $10^{-6} \mathrm{~cm}^{-1} /(\mathrm{m} / \mathrm{s})$ to reproduce for the $\mathrm{SD}_{e} \mathrm{BBP}$ fit a value of $\Delta$ consistent with recently measured collisional shifting coefficient $-3.7 \times 10^{-3} \mathrm{~cm}^{-1}$ amagat $^{-1} \cdot{ }^{70}$ Similar value was reported by Kelley and Bragg ${ }^{71}$ and is just between other experimental results. ${ }^{90,91}$

${ }^{1}$ S. Kassi and A. Campargue, J. Chem. Phys. 137, 234201 (2012). ${ }^{2}$ J. Burkart, D. Romanini and S. Kassi, Opt. Lett. in press.

${ }^{3}$ G.-W. Truong, D. A. Long, A. Cygan, D. Lisak, R. D. van Zee, and J. T. Hodges, J. Chem. Phys. 138, 094201 (2013).

${ }^{4}$ L. Moretti, A. Castrillo, E. Fasci, M. D. De Vizia, G. Casa, G. Galzerano, A. Merlone, P. Laporta, and L. Gianfrani, Phys. Rev. Lett. 111, 060803 (2013)

${ }^{5}$ C. Lemarchand, S. Mejri, P. L. T. Sow, M. Triki, S. K. Tokunaga, S. Briaudeau, C. Chardonnet, B. Darquie, and C. Daussy, Metrologia 50623 (2013). 
${ }^{6}$ A. Cygan, D. Lisak, S. Wójtewicz, J. Domysławska, J. T. Hodges, R. Trawiński, and R. Ciuryło, Phys. Rev. A. 85, 022508 (2012). ${ }^{7}$ R. H. Dicke, Phys. Rev. 89, 472 (1953).

${ }^{8}$ R. Ciuryło, Phys. Rev. A 58, 1029 (1998).

${ }^{9}$ R. Ciuryło, A. S. Pine, and J. Szudy, J. Quant. Spectrosc. Radiat. Transfer 68, 257 (2001).

${ }^{10}$ D. A. Shapiro, R. Ciuryło, R. Jaworski, and A. D. May, Can. J. Phys. 79, 1209 (2001).

${ }^{11}$ B. Lance and D. Robert, J. Chem. Phys. 109, 8283 (1998); 111, 789 (1999).

${ }^{12}$ D. Robert and L. Bonamy, Eur. Phys. J. D 2, 245 (1998).

${ }^{13}$ L. Bonamy, H. T. T. Ngoc, P. Joubert, and D. Robert, Eur. Phys. J. D 31, 459 (2004).

${ }^{14}$ H. Tran, J.-M. Hartmann, J. Chem. Phys. 130, 094301 (2009).

${ }^{15}$ J.-M. Hartmann, C. Boulet, D. Robert, Collisional Effects on Molecular Spectra (Elsevier, Amsterdam 2008).

${ }^{16}$ P. R. Berman, J. Quant. Spectrosc. Radiat. Transfer 12, 1331 (1972).

${ }^{17}$ S. G. Rautian and I. I. Sobelman, Usp. Fiz. Nauk 90, 209 (1966). [Sov. Phys. Usp. 9, 701 (1967).]

${ }^{18}$ J. Szudy, W. E. Baylis, Phys. Rep. 266, 127 (1996).

${ }^{19}$ R. Blackmore, J. Chem. Phys. 87, 791 (1987).

${ }^{20}$ R. Blackmore, S. Green, and L. Monchik, J. Chem. Phys. 91, 3846 (1989)

${ }^{21}$ A. D. May, W. -K. Liu, F. R. W. McCourt, R. Ciuryło, J. Sanchez-Fortún Stoker, D. Shapiro, and R. Wehr, Can. J. Phys. 91, 879 (2013).

${ }^{22}$ J.-M. Hartmann, H. Tran, N. H. Ngo, X. Landsheere, P. Chelin, Y. Lu, A.-W. Liu, S.-M. Hu, L. Gianfrani, G. Casa, A. Castrillo, M. Lepere, Q. Deliere, M. Dhyne, and L. Fissiaux, Phys. Rev. A 87, 013403 (2013).

${ }^{23}$ J.-M. Hartmann, V. Sironneau, C. Boulet, T. Svensson, J. T. Hodges, and C. T. Xu, Phys. Rev. A 87, 032510 (2013).

${ }^{24}$ R. Ciuryło, D. A. Shapiro, J. R. Drummond, and A. D. May, Phys. Rev. A 65, 012502 (2002).

${ }^{25}$ P. Wcisło, A. Cygan, D. Lisak, and R. Ciuryło, Phys. Rev. A 88, 012517 (2013).

${ }^{26}$ R.Wehr, A. Vitcu, R. Ciuryło, F. Thibault, J. R. Drummond, and A. D. May, Phys. Rev. A 66, 062502 (2002).

${ }^{27}$ R. Ciuryło, D. Lisak, and J. Szudy, Phys. Rev. A 66, 032701 (2002).

${ }^{28}$ R. Ciuryło, A. Bielski, J. R. Drummond, D. Lisak, A. D. May, A. S. Pine, D. A. Shapiro, J. Szudy, and R. S. Trawiński, in Spectral Line Shapes, edited by C. A. Back (AIP, Melville, NY, 2002), p. 151-160.

${ }^{29}$ D. Lisak, A. Bielski, R. Ciuryło, J. Domysławska, R. S. Trawiński, and J. Szudy, J. Phys. B: At. Mol. Opt. Phys. 36, 3985 (2003).

${ }^{30}$ R. Wehr, R. Ciuryo, A. Vitcu, F. Thibault, J. R. Drummond, and A. D. May, J. Mol. Spectrosc. 235, 54 (2006).

${ }^{31}$ R. Wehr, A. Vitcu, F. Thibault, J. R. Drummond, and A. D.May, J. Mol. Spectrosc. 235, 69 (2006).

${ }^{32}$ D. Lisak, J. T. Hodges, and R. Ciuryo, Phys. Rev. A 73, 012507 (2006).

${ }^{33}$ M. J. Lindenfeld, J. Chem. Phys. 73, 5817 (1980).

${ }^{34}$ P. F. Liao, J. E. Bjorkholm, and P. R. Berman, Phys. Rev. A 21, 1927 (1980).

${ }^{35}$ P. Wcisło, R. Ciuryło, J. Quant. Spectrosc. Radiat. Transfer 120 , 36 (2013).

${ }^{36}$ S. Chandrasekhar, Rev. Mod. Phys. 15, 1 (1943).

${ }^{37}$ D. Bohm and E. P. Gross, Phys. Rev. 75, 1851 (1949).

${ }^{38}$ L. Galatry, Phys. Rev. 122, 1218 (1961).

${ }^{39}$ M. Nelkin and A. Ghatak, Phys. Rev. 135, A4 (1964).

${ }^{40}$ P. Duggan, P. M. Sinclair, A. D. May, and J. R. Drummond, Phys. Rev. A 51, 218 (1995).

${ }^{41}$ A. Henry, D. Hurtmans, M. Margottin-Maclou, and A. Valentin, J. Quant. Spectrosc. Radiat. Transfer 56, 647 (1996).

${ }^{42}$ R. Ciuryło and J. Szudy, J. Quant. Spectrosc. Radiat. Transfer 57, 411 (1997).

${ }^{43}$ B. Lance, G. Blanquet, J. Walrand, and J.-P. Bouanich, J. Molec. Spectrosc. 185, 262 (1997).
${ }^{44}$ A. Pine, J. Quant. Spectrosc. Radiat. Transfer 62, 397 (1999).

${ }^{45}$ R. Ciuryło, R. Jaworski, J. Jurkowski, A. S. Pine, and J. Szudy, Phys. Rev. A 63, 032507 (2001).

${ }^{46}$ M. Triki, C. Lemarchand, B. Darquie, P. L. T. Sow, V. Roncin, C. Chardonnet, and C. Daussy, Phys. Rev. A 85, 062510 (2012).

${ }^{47}$ C. E. Miller, L. R. Brown, R. A. Toth, D. C. Benner, V. M. Devi, C. R. Physique 6, 876 (2005).

${ }^{48}$ C. E. Miller, D. Crisp, P. L. DeCola, S. C. Olsen, J. T. Randerson, A. M. Michalak, A. Alkhaled, P. Rayner, D. J. Jacob, P. Suntharalingam, D. B. A. Jones, A. S. Denning, M. E. Nicholls, S. C. Doney, S. Pawson, H. Boesch, B. J. Connor, I. Y. Fung, D. O'Brien, R. J. Salawitch, S. P. Sander, B. Sen, P. Tans, G. C. Toon, P. O. Wennberg, S. C. Wofsy, Y. L. Yung, R. M. Law, J. Geophys. Res. 112, D10314 (2007).

${ }^{49}$ A. Campargue, S. Kassi, K. Pachucki and J. Komasa, Phys. Chem. Chem. Phys. 14, 802 (2012).

${ }^{50}$ A. I. Burshtein and S. I. Temkin, Spectroscopy of Molecular Rotation in Gases and Liquids, (Cambridge University Press, Cambridge, 1994) 13-16.

${ }^{51}$ H. Tran, J.-M. Hartmann, F. Chaussard, and M. Gupta, J. Chem. Phys. 131, 154303 (2009).

${ }^{52}$ G. L. Rogers and P. R. Berman, J. E. M. Haverkrot, and J. P. Woerdman, Phys. Rav. A 44, 417 (1991).

${ }^{53}$ D. A. Shapiro, R. Ciuryło, J. R. Drummond, and A. D. May, Phys. Rev. A 65, 012501 (2002).

${ }^{54}$ M. J. Lindenfeld, B. Shizgal, Chem. Phys. 41, 81 (1979).

${ }^{55}$ H. Tran, F. Thibault, and J.-M. Hartmann, J. Quant. Spectrosc. Radiat. Transfer 112, 1035 (2011).

${ }^{56}$ M. P. Allen and D. J. Tildesley, Computer Simulation of Liquids (Clarendon, Oxford, 1987).

${ }^{57}$ R. C. Desai, J. Chem. Phys. 44, 77 (1966).

${ }^{58}$ P. N. M. Hoang, P. Joubert, and D. Robert, Phys. Rev. A 65, 012507 (2002).

${ }^{59}$ G. Audi, A.H. Wapstra and C. Thibault, Nucl. Phys. A 729, 337 (2003).

${ }^{60}$ B. J. Alder and T. E. Wainwright, Phys. Rev. Lett. 18, 988 (1967).

${ }^{61}$ B. J. Alder and T. E. Wainwright, Phys. Rev. A 1, 18 (1970).

${ }^{62}$ H. Tran, N. H. Ngo, J.-M. Hartmann, R. R. Gamache, D. Mondelain, S. Kassi, A. Campargue, L. Gianfrani, A. Castrillo, E. Fasci and F. Rohart, J. Chem. Phys. 138, 034302 (2013).

${ }^{63}$ L. S. Rothman, I. E. Gordon, A. Barbe, D. Chris Benner, P. F. Bernath, M. Birk, V. Boudon, L. R. Brown, A. Campargue, J.-P. Champion, K. Chance, L. H. Coudert, V. Dana, V. M. Devi, S. Fally, J.-M. Flaud, R. R. Gamache, A. Goldman, D. Jacquemart, I. Kleiner, N. Lacome, W. J. Lafferty, J.-Y. Mandin, S. T. Massie, S. N. Mikhailenko, C. E. Miller, N. Moazzen-Ahmadi, O. V. Naumenko, A. V. Nikitin, J. Orphal, V. I. Perevalov, A. Perrin, A. Predoi-Cross, C. P. Rinsland, M. Rotger, M. Šimečková, M. A. H. Smith, K. Sung, S. A. Tashkun, J. Tennyson, R. A. Toth, A. C. Vandaele, and J. Vander Auwera, J. Quant. Spectrosc. Radiat. Transfer 110, 533 (2009).

${ }^{64}$ E. W. Smith, J. Cooper, W. R. Chappell, and T. Dillon, J. Quant. Spectrosc. Radiat. Transfer 11, 1547 (1971); 11, 1567 (1971).

${ }^{65}$ G. Nienhuis, J. Quant. Spectrosc. Radiat. Transfer 20, 275 (1978).

${ }^{66}$ A.S. Pine, J. Chem. Phys. 101, 3444 (1994).

${ }^{67}$ L. Demeio, S. Green, L. Monchick, J. Chem. Phys. 102, 9160 (1995).

${ }^{68}$ J. Ward, J. Cooper, E.W. Smith, J. Quant. Spectrosc. Radiat. Transfer 14, 555 (1974).

${ }^{69}$ J. W. Forsman, J. Bonamy, and D. Robert, J. Ph. Berger, R. Saint-Loup, and H. Berger, Phys. Rev. A 52, 2652 (1995).

${ }^{70}$ S. Kassi and A. Campargue, J. Mol. Spectrosc. 300, 55 (2014).

${ }^{71}$ J. D. Kelley, S. L. Bragg, Phys. Rev. A 34, 3003 (1986).

${ }^{72}$ F. Rohart, H. Mader, H.-W. Nicolaisen, J. Chem. Phys. 101, 6475 (1994)

${ }^{73}$ J. Tennyson, P. F, Bernath, A. Campargue, A. G. Csaszar, L. Daumont, R. R. Gamache, J. T Hodges, D. Lisak, O. V. Naumenko, L. S. Rothman, H. Tran, N. F. Zobov, J. Buldyreva, C. 
D. Boone, M. D. De Vizia, L. Gianfrani, J.-M. Hartmann, R. McPheat, D. Weidmann, J. Murray, N. H. Ngo, O. L. Polyansky, Pure Appl. Chem. (2014) accepted.

${ }^{74}$ N. H. Ngo, D. Lisak, H. Tran, J.-M. Hartmann, J. Quant. Spectrosc. Radiat. Transfer 129, 89 (2013)

${ }^{75}$ N. H. Ngo, D. Lisak, H. Tran, J.-M. Hartmann, J. Quant. Spectrosc. Radiat. Transfer 134, 105 (2014).

${ }^{76}$ H. Tran, N. H. Ngo, J.-M. Hartmann, J. Quant. Spectrosc. Radiat. Transfer 129, 199 (2013).

${ }^{77}$ H. Tran H, N. H. Ngo, J.-M, Hartmann, J. Quant. Spectrosc. Radiat. Transfer 134, 104 (2014).

${ }^{78}$ A. Cygan, S. Wójtewicz, J. Domysławska, P. Masłowski, K. Bielska, M. Piwiński, K. Stec, R. S. Trawiński, F. Ozimek, C. Radzewicz, H. Abe, T. Ido, J. T. Hodges, D. Lisak, R. Ciuryło, Eur. Phys. J.- Spec. Top. 222, 2119 (2013).

${ }^{79}$ F. Chaussard, X. Michaut, R. Saint-Loup, H. Berger, P. Joubert, B. Lance, J. Bonamy, D. Robert, J. Chem. Phys. 112, 158 (2000).

${ }^{80}$ F. Chaussard, R. Saint-Loup, H. Berger, P. Joubert, X. Bruet, J. Bonamy, D. Robert, J. Chem. Phys. 113, 4951 (2000).

${ }^{81} \mathrm{~S}$. Wójtewicz, A. Cygan, P. Masłowski, J. Domysławska, D. Lisak, R. S. Trawiński, R. Ciuryło, J. Quant. Spectrosc. Radiat. Transfer 144, 36 (2014).

${ }^{82}$ P. J. Bendt, Phys. Rev. 110, 85 (1958).

${ }^{83}$ J. O. Hirschfelder, C. F. Curtis, and R. B. Bird, Molecular Theory of Gases and Liquids (Wiley, New York, 1954) 578-581.

${ }^{84}$ D. C. Benner, C. P. Rinsland, V. Malathy Devi, M. A. H. Smith, D. Atkins, J. Quant. Spectrosc. Radiat. Transfer 53, 705 (1995).
${ }^{85}$ J. Komasa, K. Piszczatowski, G. Łach, M. Przybytek, B. Jeziorski and K. Pachucki, J. Chem. Theory Comput. 7, 3105 (2011).

${ }^{86}$ A. Cygan, D. Lisak, R. S. Trawiński, and R. Ciuryło, Phys. Rev. A 82, 032515 (2010).

${ }^{87}$ T. I. Ivanov, M. Roudjane, M. O. Vieitez, C. A. de Lange, W.-Ü L. Tchang-Brillet, and W. Ubachs Phys. Rev. Lett. 100, 093007 (2008).

${ }^{88}$ E. J. Salumbides, J. C. J. Koelemeij, J. Komasa, K. Pachucki, K. S. E. Eikema, and W. Ubachs, Phys. Rev. D 87, 112008 (2013).

${ }^{89}$ L. S. Rothman, I. E. Gordon, Y. Babikov, A. Barbe, D. Chris Benner, P. F. Bernath, M. Birk, L. Bizzocchi, V. Boudon, L. R. Brown, A. Campargue, K. Chance, E. A. Cohen, L. H. Coudert, V. M. Devi, B. J. Drouin, A. Fayt, J.-M. Flaud, R. R. Gamache, J. J. Harrison, J.-M. Hartmann, C. Hill, J. T. Hodges, D. Jacquemart, A. Jolly, J. Lamouroux, R. J. Le Roy, G.Li, D. A. Long, O. M. Lyulin, C. J. Mackie, S. T. Massie, S. Mikhailenko, H. S. P. Mller, O. V. Naumenko, A. V. Nikitin, J. Orphal, V. Perevalov, A. Perrin, E. R. Polovtseva, C. Richard, M. A. H. Smith, E. Starikova, K. Sung, S. Tashkun, J. Tennyson, G. C. Toon, Vl. G. Tyuterev, G. Wagner, J. Quant. Spectrosc. Radiat. Transfer 130, 4 (2013).

${ }^{90}$ S. L. Bragg, J. W. Brault and W. H. Smith, Astrophys. J. 263, 999 (1982).

${ }^{91}$ M. Gupta, T. Owano, D. S. Baer and A. O'Keefe, Chem. Phys. Lett. 418, 11 (2006). 